\title{
ÉTUDE SUR LES VARIATIONS \\ DES TENEURS EN MATIÈRES AZOTÉES ET EN ÉLÉMENTS MINÉRAUX DU DACTYLE
}

PAR

\section{GUEGUEN et G. FA UCONNEAU}

Avec la collaboration technique de Valérie Françors-Goujon et de B. Teilhard de Chardin. Service de Biochimie et de Nutrition, C. N. R. Z., Jouy-en-Josas (S.-et-O.).

\section{SOMMAIRE}

Les variations des teneurs en matières azotées et en éléments minéraux $(\mathrm{P}, \mathrm{Ca}, \mathrm{K}, \mathrm{Na}, \mathrm{Mg}$ ) d'une graminée fourragère, le dactyle, ont été étudiées après séparation de la plante en différentes parties : limbes, gaines, tiges, fleurs et débris ; ceci a également permis de suivre l'évolution de la composition de ces divers organes.

Les parcelles expérimentales ont été subdivisées de manière à obtenir plusieurs cycles de végétation provenant du cycle précédent interrompu à des stades de développement variables, ce qui a permis d'étudier les avantages ou les inconvénients de divers modes d'exploitation. Durant chacun des cycles étudiés, le dactyle a été récolté à trois stades de développement différents.

Les matières azotées $(\mathrm{N} \times 6,25)$ ont été dosées par la méthode de Kjeldahl. Les éléments minéraux ont été dosés après calcination du produit : le phosphore par une méthode colorimétrique au phosphovanadomolybdate d'ammonium, et le calcium, le sodium, le potassium et le magnésium par spectrophotométrie de flamme.

En règle générale, le premier cycle de végétation diffère profondément des cycles suivants, d'une part quant à la composition chimique même de la plante et de ses divers organes, d'autre part quant à l'évolution de cette composition avec l'âge de la plante. Ces différences sont dues en premier lieu aux dissemblances morphologiques entre les plantes, puisque généralement la montaison et la floraison ne se produisent plus après le premier cycle.

Les différences de teneurs entre organes, importantes au premier cycle, où les limbes sont en général les plus riches, deviennent négligeables au cours des cycles suivants, où limbes et gaines ont des compositions voisines, mis à part les matières azotées.

A stade de développement équivalent, les plantes des derniers cycles sont en général les mieux pourvues en $\mathrm{P}, \mathrm{Ca}, \mathrm{Na}, \mathrm{Mg}$, dont les taux augmentent régulièrement au cours de l'année. En revanche le potassium est plus abondant 
dans les plantes du premier cycle, et son taux diminue au cours des cycles suivants.

L'influence du stade de développement sur les teneurs du dactyle en matières azotées, calcium et magnésium est très nette, quel que soit le cycle de végétation : celles-ci diminuent régulièrement avec l'âge de la plante. En revanche, pour le phosphore et le potassium, seules les plantes du premier cycle s'appauvrissent considérablement, la composition des plantes des cycles suivants étant peu modifiée par le stade de développement. Ceci est particulièrement important pour la teneur en phosphore de la plante, qui ne diminue considérablement qu'au cours du premier cycle, phénomène que l'on a souvent tendance à généraliser aux cycles suivants. Enfin, l'âge de la plante influe de façon très irrégulière sur sa teneur en sodium, qui, dans le dactyle étudié, est particulièrement élevée à partir du second cycle.

La comparaison des besoins des animaux en chacun des éléments étudiés aux teneurs trouvées dans les plantes des différents cycles, a permis, compte tenu des variations du rendement en matière sèche, de définir le mode d'exploitation à préconiser.

La connaissance de la composition chimique des fourrages demeure très imparfaite, et peu de travaux ont été effectués sur des espèces pures de plantes fourragères, dans le but d'étudier systématiquement l'influence du stade de développement et du cycle de végétation sur la composition chimique de la plante entière et de ses divers organes.

Nous avons donc entrepris une étude systématique de la composition minérale d'une graminée fourragère fréquemment utilisée seule, le dactyle, en comparant plusieurs cycles successifs de végétation, et en étudiant l'influence du stade d'interruption de chaque cycle sur la composition des repousses. Cette expérience complète un travail antérieur comportant seulement l'étude du premier cycle de végétation d'un dactyle et d'une fétuque (GuEGuen I959), et précède un essai analogue actuellement en cours sur la fétuque des prés.

\section{I. - MATÉRIEL E'T MÉTHODES}

\section{Matériel expérimental.}

L'expérience porte sur un dactyle S 26 semé au printemps I957 sur une parcelle du C.N. R. Z. (sol argilo-humifère), fumé abondamment et récolté durant l'année I958 (après une exploitation normale en r957) à trois stades de développement durant plusieurs cycles de végétation. Les trois stades de récolte choisis correspondent pour le premier cycle respectivement au stade feuillu, à l'épiaison et à la floraison, et pour les cycles suivants à trois durées de croissance : 4 semaines, 6 semaines et 8 semaines. Les différentes parcelles sont subdivisées après chaque coupe de manière à pouvoir obtenir des cycles de végétation ultérieurs provenant 
(9, I960) MATIÈRES AZOTÉES ÉT ÉLÉMENTS MINÉRAUX

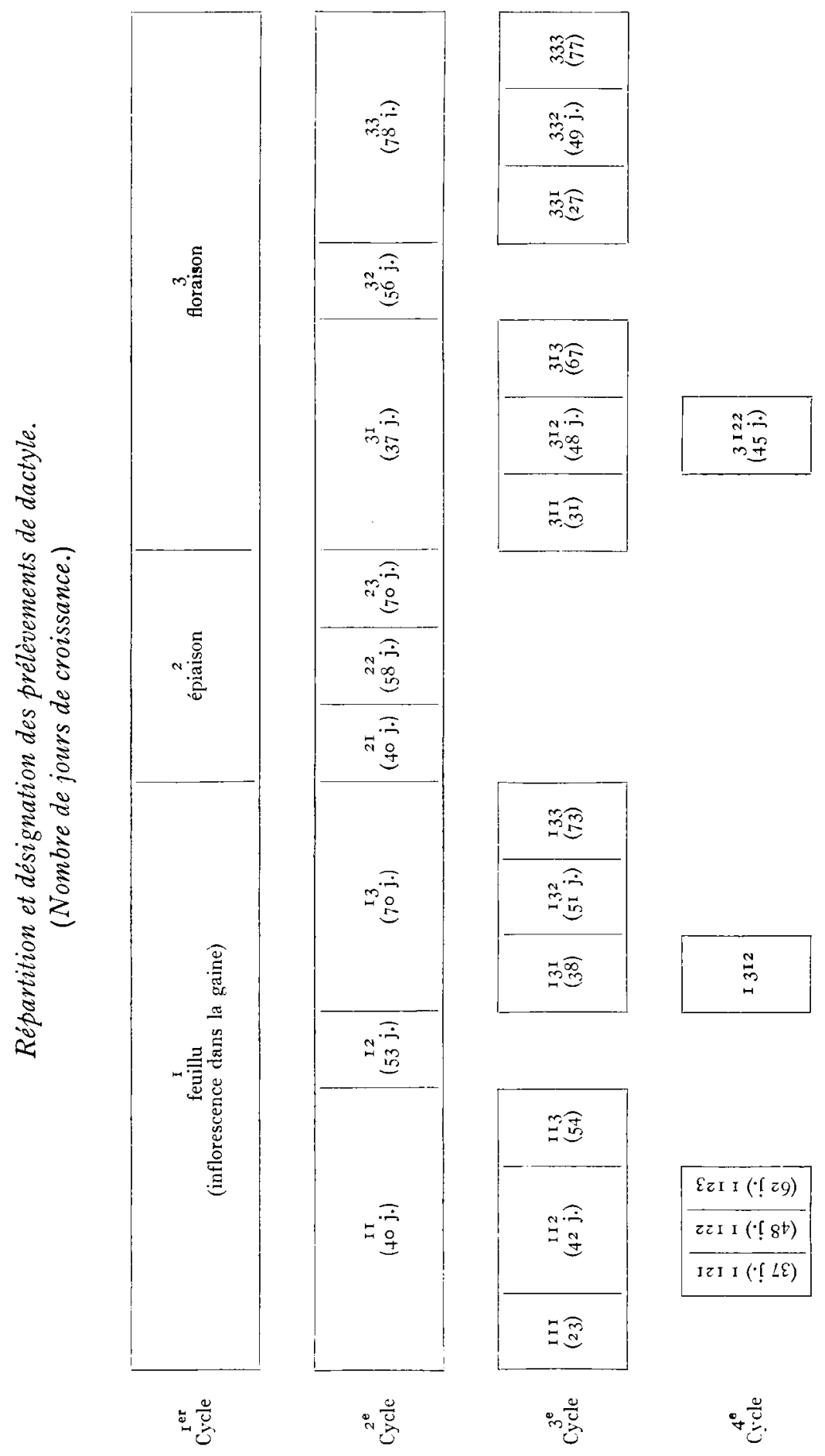


d'une coupe précoce (25-35 jours de croissance), normale (40-5o j) ou tardive $(60-70$ j) du cycle précédent. Le tableau suivant indique la répartition des différentes coupes et la durée de croissance correspondante (en jours). La nomenclature des coupes (également utilisée dans les graphiques et tableaux) permet de connaître le numéro d'ordre du cycle de végétation (nombre total de chiffres), le stade de développement (chiffre des unités) et la ou les coupes antérieures (chiffres précédant le chiffre des unités) (cf. tableau et exemple ( ${ }^{1}$ ).

\section{Mode de prélèvement et traitement des échantillons.}

Un échantillon représentatif pesant environ $3 \mathrm{~kg}$ est prélevé au hasard sur la totalité de la parcelle fauchée (Io $\mathrm{m}^{2}$ au minimum) et la production par mètre carré est calculée en poids de matière fraîche

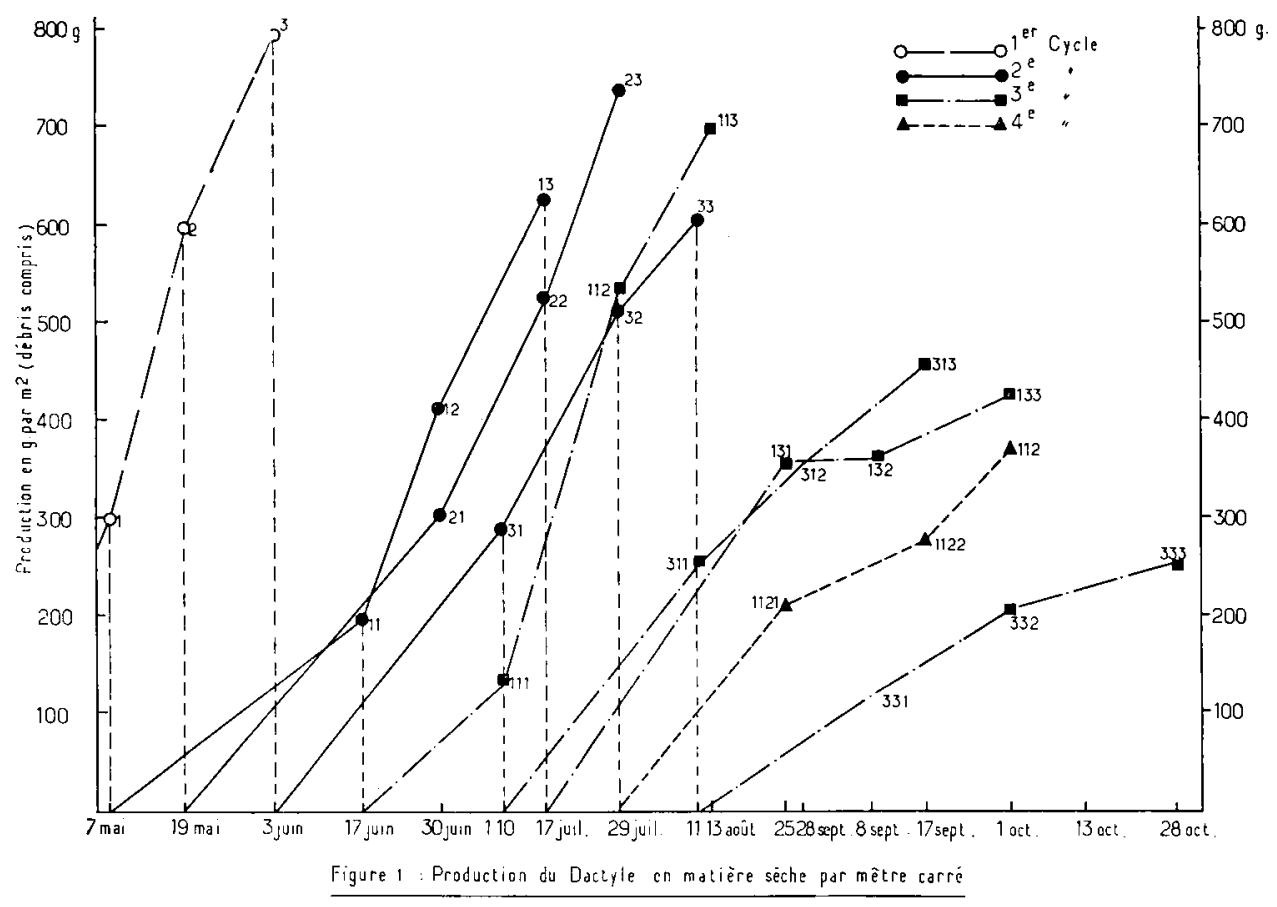

et de matière sèche (figure I). Les limbes, gaines, tiges, fleurs et éventuellement débris et plantes adventices sont minutieusement séparés, et l'on détermine leurs proportions relatives en poids de matière sèche après passage au four à circulation d'air $\left(60-75^{\circ} \mathrm{C}\right)$.

Les échantillons sont finement broyés et conservés en chambre froide à $0-2^{\circ} \mathrm{C}$.

(1) Exemple : la coupe numérotée 132 a été récoltée au ze stade de développement (équivalent à 6 semaines de croissance), fait partie d'un 3 e cycle de végétation, repousse d'une coupe effectuée

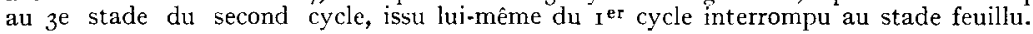




\section{Méthodes d'analyse.}

Les méthodes de dosage utilisées ont été choisies en raison de leur rapidité et de leur bonne fidélité, et ont fait l'objet de mises an point préalables (GUeguen et Rombauts 1960).

Les matières azotées $(N \times 6,25)$ sont obtenues par la méthode de Kjeldahl.

Les éléments minéraux étudiés sont dosés dans le résidu de calcination pendant 8 à Io heures, à $530^{\circ} \mathrm{C}$, d'une prise d'essai de I g de produit. I es cendres sont reprises par de l'acide chlorhydrique pur, sur bain de sable, pour insolubiliser la silice, puis l'hydrolyse (nécessaire pour la transformation des pyrophosphates éventuellement formés en orthophosphates).est effectuée en reprenant par un peu d'acide nitrique dilué et en maintenant à l'ébullition durant 5 minutes. Après filtration on complète à Ioo $\mathrm{ml}$ avec de l'eau bi-distillée.

Le phosphore est dosé par la méthode de Misson au phosphovanadomolybdate d'ammonitum. L'intensité de la coloration jaune obtenue par 1'addition d'un réactif nitro-vanado-molybdique, est mesurée au spectrophotomètre à $430 \mathrm{~m} \mu$. I a gamme de solutions étalons utilisées s'étend de 5 à $40 \gamma$ de $\mathrm{P}$ par $\mathrm{mll}$.

L e sodium, le potassium, le calcium et le magnésinm sont dosés par spectrophotométrie de flamme, à l'aide du photomètre à flamme « Éppendorf " par passage direct de la solution précédemment obtenue. L'étalonnage de l'appareil est effectué à l'aide de solutions témoins contenant l'élément à doser à des concentrations convenables (en général de Io à 5 o $\curlyvee$ par m1) en solution pure ou en tnélange, compte tenu des principales interférences observées ( $\mathrm{K}$ sur $\mathrm{Na}, \mathrm{K}$ et $\mathrm{Na}$ sur $\mathrm{Mg}$ ).

$\mathrm{Na}$ et $\mathrm{K}$ sont dosés dans la flamme air-propane, Ca dans la flamme air-acétylène et $\mathrm{Mg}$ dans la flamme air-hydrogène-oxygène.

Tous les chiffres cités sont les moyennes de deux dosages effectués sur des prises différentes, l'écart maximum admis entre les doubles étant de 5 p. Ioo.

\section{II. - RÉSULTATS}

\section{Interprétation du point de vue de la physiologie végétale.}

Dans l'exposé de nos résultatis nous comparerons les cycles de végétation successifs, d'une part du point de vue des teneurs de la plante et de ses divers organes en éléments minéraux, et d'autre part du point de vue de l'évolution de ces teneurs en fonction du stade de développement de la plante.

Il importe tout d'abord de remarquer que la plupart des différences 
de composition observées entre les cycles dépendent en premier lieu des dissemblances morphologiques fondamentales qui existent entre les plantes du I ${ }^{\text {er }}$ cycle et les plantes des cycles suivants. En effet, le premier cycle complet du dactyle aboutit à la floraison, après la montaison et l'épiaison, en produisant une abondance de tiges, tandis que les cycles suivants, provenant d'une première coupe effectuée à la montaison ou à l'épiaison (les plantes étant sectionnées plus bas que l'épi), sont presque exclusivement constituées de limbes et de gaines dont la proportion varie peu avec l'âge ( 80 à 90 p. roo).

\section{TABLEAU I}

Pourcentages, en poids de matière sèche, des différentes parties de la plante par rapport à la plante entière.

$$
\begin{array}{lll}
\mathrm{L}=\text { Limbes } & \mathrm{T}=\text { Tiges } & \mathrm{D}=\text { Débris } \\
\mathrm{G}=\text { Gaines } & \mathrm{F}=\text { Fleurs } & \mathrm{P} \mathrm{E}=\text { Plante entière }
\end{array}
$$

\begin{tabular}{|c|c|c|c|c|c|c|c|c|}
\hline Coupe & Organe & $\%$ & Coupe & Organe & $\%$ & Coupe & Organe & $\%$ \\
\hline I & $\frac{\mathrm{L}}{\mathrm{G}}+\mathrm{T}$ & $\begin{array}{l}45,84 \\
41,96 \\
\mathrm{I} 2,20\end{array}$ & $3 \mathrm{I}$ & $\begin{array}{l}\mathrm{L} \\
\mathrm{G} \\
\mathrm{D}\end{array}$ & $\begin{array}{r}80,14 \\
16,64 \\
3,22\end{array}$ & 312 & $\begin{array}{l}\mathrm{L} \\
\mathrm{G} \\
\mathrm{D}\end{array}$ & $\begin{array}{l}56,65 \\
\text { I } 5,76 \\
27,58\end{array}$ \\
\hline 2 & $\begin{array}{l}\mathrm{L} \\
\mathrm{G} \\
\mathrm{T}\end{array}$ & $\begin{array}{l}26,66 \\
27,18 \\
3 \mathrm{I}, 28\end{array}$ & 32 & $\begin{array}{l}\mathrm{L} \\
\mathrm{G} \\
\mathrm{D}\end{array}$ & $\begin{array}{l}66,82 \\
14,22 \\
18,96\end{array}$ & $3 \mathrm{I} 3$ & $\begin{array}{l}\mathrm{L} \\
\mathrm{G} \\
\mathrm{D}\end{array}$ & $\begin{array}{l}48,5 \mathrm{I} \\
\mathrm{I} 2,49 \\
38,98\end{array}$ \\
\hline 3 & $\stackrel{\mathrm{L}}{\mathrm{G}}+\mathrm{T}$ & $\begin{array}{l}r 0,73 \\
65,41\end{array}$ & 33 & $\begin{array}{l}\mathrm{L} \\
\mathrm{G} \\
\mathrm{D}\end{array}$ & $\begin{array}{l}51,8,3 \\
15,89 \\
32,27\end{array}$ & $33^{\mathrm{I}}$ & $\begin{array}{l}L \\
G \\
D\end{array}$ & $\begin{array}{r}76,18 \\
9,72 \\
14,10\end{array}$ \\
\hline J & & $\begin{array}{r}17,32 \\
6,54 \\
\end{array}$ & II I & $\underset{G}{\mathrm{~L}}$ & $\begin{array}{l}81,6,3 \\
\mathrm{I} 3,12\end{array}$ & 332 & $\underset{G}{\mathrm{~L}}$ & $\begin{array}{l}68,10 \\
11,57\end{array}$ \\
\hline & $\mathrm{I}$ & 79,55 & & D & 5,25 & & D & 20,33 \\
\hline I I & $\mathrm{G}^{\mathrm{G}}+\mathrm{T}$ & $\begin{array}{r}16,22 \\
4,23\end{array}$ & $\mathrm{II} 2$ & $\mathrm{~L}$ & $\begin{array}{l}70,92 \\
13,67\end{array}$ & 333 & $\mathrm{~L}$ & $\begin{array}{r}55,44 \\
I, 29\end{array}$ \\
\hline & I & 74,73 & & $\mathrm{D}$ & I5,38 & & D & 30,27 \\
\hline 12 & $\begin{array}{l}G+T \\
F \\
D\end{array}$ & $\begin{array}{r}19,49 \\
0,79 \\
5,05\end{array}$ & $\mathrm{II}_{3}$ & $\begin{array}{l}\mathrm{L} \\
\mathrm{G} \\
\mathrm{D}\end{array}$ & $\begin{array}{l}48,58 \\
17,32 \\
34,10\end{array}$ & I I 21 & $\begin{array}{l}\mathrm{L} \\
\mathrm{G} \\
\mathrm{D}\end{array}$ & $\begin{array}{r}76,34 \\
9,78 \\
13,88\end{array}$ \\
\hline 13 & $\begin{array}{l}\mathrm{L} \\
\mathrm{G} \\
\mathrm{D}\end{array}$ & $\begin{array}{l}63,71 \\
21,70 \\
14,58 \\
-\end{array}$ & I3I & $\begin{array}{l}\mathrm{L} \\
\mathrm{G} \\
\mathrm{D}\end{array}$ & $\begin{array}{r}82,62 \\
11,09 \\
6,29\end{array}$ & I I 22 & $\begin{array}{l}\mathrm{L} \\
\text { (; } \\
\mathrm{D}\end{array}$ & $\begin{array}{l}55,75 \\
\text { I3, IO } \\
3 \text { I, } 15\end{array}$ \\
\hline $2 \mathrm{I}$ & $\begin{array}{l}\mathrm{L} \\
\mathrm{G} \\
\mathrm{D}\end{array}$ & $\begin{array}{r}8 \mathrm{r}, 75 \\
\mathrm{I} 6,99 \\
\mathrm{I}, 25\end{array}$ & ${ }^{1} 3^{2}$ & $\begin{array}{l}\mathrm{L} \\
\mathrm{G} \\
\mathrm{D}\end{array}$ & $\begin{array}{l}64,05 \\
15,03 \\
20,9 \mathrm{I}\end{array}$ & I 123 & $\begin{array}{l}\mathbf{L} \\
G \\
\mathbf{D}\end{array}$ & $\begin{array}{l}51,84 \\
15,48 \\
32,68\end{array}$ \\
\hline 22 & $\begin{array}{l}\mathrm{I} \\
\mathrm{G} \\
\mathrm{D}\end{array}$ & $\begin{array}{l}66,05 \\
20,79 \\
13,15\end{array}$ & I33 & $\begin{array}{l}\mathrm{L} \\
\mathrm{G} \\
\mathrm{D}\end{array}$ & $\begin{array}{l}51,65 \\
15,29 \\
33,06\end{array}$ & I 352 & $\begin{array}{l}\mathrm{L} \\
\mathrm{Cr} \\
\mathrm{D}\end{array}$ & $\begin{array}{r}57,92 \\
8,75 \\
33,33\end{array}$ \\
\hline 23 & $\begin{array}{l}\mathrm{I} \\
\mathrm{G} \\
\mathrm{D}\end{array}$ & $\begin{array}{l}55,78 \\
17,77 \\
26,45\end{array}$ & $3^{\text {I I }}$ & $\begin{array}{l}\mathrm{L} \\
\mathrm{G} \\
\mathrm{D}\end{array}$ & $\begin{array}{l}72,77 \\
15,18 \\
12,04\end{array}$ & 3122 & $\begin{array}{l}\mathrm{L} \\
\mathrm{G} \\
\mathrm{D}\end{array}$ & $\begin{array}{l}46,54 \\
10,55 \\
42,90\end{array}$ \\
\hline
\end{tabular}


Ënfin, notre travail ne portant que sur la partie saine et consommable de la plante, nous avons éliminé les extrémités rouillées des limbes, souvent abondantes en automne, et refusées par les animaux (cf. tableau I). C'est pourquoi notre étude, en rapport avec la nutrition animale, ne peut se comparer en tout point à d'autres travaux de physiologie végétale.

\section{$1^{\circ}$ Les Matières Azotées.}

\section{Composition des divers organes et de la plante entière.}

Les limbes sont toujours plus riches ( 2 à 3 fois) que les gaines dans tous les cycles (tableau II). I a diminution des teneurs en matières azotées des limbes (qui se produit à tous les cycles) est liée à leur croissance :

- Au cours du premier cycle, la croissance des limbes a lieu avant 1'épiaison; aussi la diminution des teneurs en matières azotées se fait précocement et les limbes du $\mathrm{I}^{\mathrm{er}}$ cycle sont plus pauvres.

- Au cours des cycles suivants, les conditions climatiques plus ou moins favorables permettent des croissances plus ou moins longues et la teneur minimum ( 6 p. Ioo) est atteinte d'autant plus vite que les conditions climatiques sont défavorables. En I 958 l'été pluvieux a permis la croissance des limbes (des $2^{\mathrm{e}} \cdot 3^{\mathrm{e}}$ etc. cycles) pendant toute la saison, ce qui a contribué à maintenir des teneurs élevées; en outre, celles-ci sont artificiellement augmentées au cours des derniers cycles par suite des proportions importantes de limbes rouillés éliminés (les analyses n'ont porté que sur les limbes sains, c'est-à-dire les plus jeunes) (figure II).

\section{Influence du stade de développement.}

Les teneurs en matières azotées des gaines et des tiges évoluent très vite au cours du premier cycle de végétation, l'azote étant dilué par les glucides membranaires qui se forment en abondance au cours de l'allongement des tiges (montaison). I,es teneurs en constituants membranaires et en matières azotées sont en effet inversement proportionnelles, puisque ces dernières constituent un élément important du protoplasme localisé dans les cellules vivantes des parenchymes chlorophylliens.

Au cours des cycles suivants, la teneur des gaines diminue régulièrement avec l'âge et les différents cycles se ressemblent; cependant les coupes tardives tendent à produire des gaines s'appauvrissant plus vite (Io-7,56 p. Ioo pour les échantillons 3 I 3 I-3I $32-3$ I 33 et II-6, 25 p. Ioo pour les échantillons 33I-332-33).

I'évolution des teneurs en matières azotées de la plante entière 
TABLEAU II

Évolution de la teneur en matières azotées $(N \times 6,25)$ du dactyle. En g par $100 \mathrm{~g}$ de matière sèche.

\begin{tabular}{|c|c|c|c|c|c|c|c|c|}
\hline Coupe & Organe & M. A. & Coupe & Organe & M. A. & Coupe & Organe & M. A. \\
\hline I & $\begin{array}{l}\mathrm{I} \\
\mathrm{C} \\
\mathrm{PE}\end{array}$ & $\begin{array}{r}17,69 \\
9,44 \\
13,74\end{array}$ & $3 \mathrm{I}$ & $\begin{array}{l}\mathrm{L} \\
\mathrm{G} \\
\mathrm{PL}\end{array}$ & $\begin{array}{l}22,19 \\
10,38 \\
20,15\end{array}$ & $3^{12}$ & $\begin{array}{l}\mathrm{L} \\
\mathrm{G} \\
\mathrm{PE}\end{array}$ & $\begin{array}{l}21,38 \\
10,88 \\
19,09\end{array}$ \\
\hline 2 & $\begin{array}{l}\mathrm{L} \\
G \\
T \\
\mathrm{~F}\end{array}$ & $\begin{array}{r}16,63 \\
5,63 \\
4,50 \\
12,31\end{array}$ & 32 & $\begin{array}{l}\mathrm{I} \\
G \\
\mathrm{P} \mathrm{L}\end{array}$ & $\begin{array}{r}18,44 \\
8,13 \\
16,63\end{array}$ & $3^{1} 3$ & $\begin{array}{l}\mathrm{L} \\
\mathrm{G} \\
\mathrm{PE}\end{array}$ & $\begin{array}{r}20,12 \\
9,37 \\
17,92 \\
\end{array}$ \\
\hline & PE & 9,20 & 3.3 & $\begin{array}{l}\mathrm{L} \\
\mathrm{G} \\
\mathrm{P}^{\prime} \mathrm{E}\end{array}$ & $\begin{array}{r}17,13 \\
7,56 \\
14.88\end{array}$ & $33^{\mathrm{I}}$ & $\begin{array}{l}\mathrm{L} \\
\mathrm{G} \\
\mathrm{PE}\end{array}$ & $\begin{array}{l}2,3,12 \\
11,0 \\
21,74\end{array}$ \\
\hline 3 & $\begin{array}{l}\mathrm{L} \\
\mathbf{G}+\mathrm{T} \\
\mathrm{F} \\
\mathrm{P} \mathrm{E}\end{array}$ & $\begin{array}{r}15,06 \\
3,63 \\
10,75 \\
6,26 \\
\end{array}$ & III & $\begin{array}{l}\mathrm{L} \\
\text { (; } \\
\text { PE: }\end{array}$ & $\begin{array}{l}25,75 \\
12,81 \\
2,3,95 \\
\end{array}$ & $33^{2}$ & $\begin{array}{l}\mathrm{L} \\
\mathrm{G} \\
\mathrm{PE}\end{array}$ & $\begin{array}{r}21,74 \\
16,18 \\
6,25 \\
14,74 \\
\end{array}$ \\
\hline I I & $\begin{array}{l}\mathrm{L} \\
\mathrm{G}+\mathrm{T} \\
\mathrm{F} \\
\mathrm{PE}\end{array}$ & $\begin{array}{l}26, \mathrm{I}_{3} \\
10,88 \\
\mathrm{II}, 75 \\
23,03\end{array}$ & II 2 & $\begin{array}{l}\mathrm{L} \\
\mathrm{G} \\
\mathrm{PE}\end{array}$ & $\begin{array}{r}19,75 \\
9,56 \\
18,10\end{array}$ & 333 & $\begin{array}{l}\mathrm{L} \\
\mathrm{G} \\
\mathrm{PE}\end{array}$ & $\begin{array}{r}17,94 \\
7,50 \\
15,80\end{array}$ \\
\hline 12 & $\begin{array}{l}\mathrm{L} \\
\mathrm{G} \\
\mathrm{P} E\end{array}$ & $\begin{array}{r}21,69 \\
9,81 \\
18,84\end{array}$ & $\mathrm{II}_{3}$ & $\begin{array}{l}\mathrm{L} \\
\mathrm{G} \\
\mathrm{P}^{\prime} \mathrm{I}\end{array}$ & $\begin{array}{l}r 9,63 \\
10,88 \\
r 7,33\end{array}$ & I I I I & $\begin{array}{l}\mathrm{L} \\
\mathrm{G} \\
\mathrm{PE}\end{array}$ & $\begin{array}{l}25,8 \mathrm{I} \\
1,3,3 \mathrm{I} \\
24,39\end{array}$ \\
\hline 13 & $\begin{array}{l}\mathrm{L} \\
\mathrm{G} \\
\mathrm{P} \mathrm{E}\end{array}$ & $\begin{array}{r}17,25 \\
9,3 \mathrm{I} \\
15,23\end{array}$ & $13 \mathrm{I}$ & $\begin{array}{l}\mathrm{L} \\
\mathrm{G} \\
\mathrm{Pl}\end{array}$ & $\begin{array}{l}26,37 \\
1,3,25 \\
24,82\end{array}$ & I 122 & $\begin{array}{l}\mathrm{L} \\
\mathrm{G} \\
\mathrm{PE}\end{array}$ & $\begin{array}{l}23,25 \\
11,50 \\
21,01\end{array}$ \\
\hline $2 I$ & $\begin{array}{l}\mathrm{L} \\
\mathrm{G} \\
\mathrm{PE}\end{array}$ & $\begin{array}{r}21,69 \\
9,81 \\
19,65\end{array}$ & ${ }^{1} 32$ & $\begin{array}{l}\mathrm{L} \\
\mathrm{G} \\
\mathrm{PL}\end{array}$ & $\begin{array}{l}25,25 \\
12,62 \\
22,85\end{array}$ & I 123 & $\begin{array}{l}\mathrm{L} \\
\mathrm{G} \\
\mathrm{PE}\end{array}$ & $\begin{array}{r}20,50 \\
9,12 \\
17,88\end{array}$ \\
\hline 22 & $\begin{array}{l}\mathrm{L} \\
\mathrm{G} \\
\mathrm{PE}\end{array}$ & $\begin{array}{r}16,94 \\
8,69 \\
\mathbf{1 4 , 9 6}\end{array}$ & $I_{33}$ & $\begin{array}{l}\mathrm{L} \\
\mathrm{G} \\
\mathrm{PE}\end{array}$ & $\begin{array}{l}21,50 \\
10,18 \\
18,91\end{array}$ & I 3 I 2 & $\begin{array}{l}\mathrm{L} \\
\mathrm{G} \\
\mathrm{PE}\end{array}$ & $\begin{array}{l}30,12 \\
12,94 \\
27,86\end{array}$ \\
\hline 2.3 & $\begin{array}{l}\mathrm{L} \\
\mathbf{G} \\
\mathrm{P} \mathrm{E}\end{array}$ & $\begin{array}{r}16,56 \\
7,69 \\
20,15\end{array}$ & $3 \mathrm{II}$ & $\begin{array}{l}\mathrm{L} \\
\mathrm{G} \\
\mathrm{PE}\end{array}$ & $\begin{array}{l}22,56 \\
12,06 \\
20,74\end{array}$ & 3122 & $\begin{array}{l}\mathrm{L} \\
\mathrm{G} \\
\mathrm{PE}\end{array}$ & $\begin{array}{l}22,19 \\
11,37 \\
20,19\end{array}$ \\
\hline
\end{tabular}

résulte essentiellement des variations morphologiques au cours des premiers cycles (le rapport limbes/plante entière diminue de 46 à II p. IOO), et de l'évolution des différents organes au cours des cycles suivants toujours feuillus (rapport limbes/plante entière varie de 80 à $90 \mathrm{p}$. Ioo).

\section{$2^{\circ}$ Le Phosphore.}

Compositon des divers organes et de la plante entière.

Au cours du premier cycle de végétation, nous constatons de nouveau (GUEGUEN I959) que les limbes sont plus riches que les gaines et les tiges, du moins pour les plantes âgées. Pendant les cycles suivants les 
gaines demeurent un peu moins riches que les limbes, la différence étant parfois peu marquée.

A stade botanique équivalent, limbes et gaines s'enrichissent très nettement en $\mathrm{P}$ au cours de l'année (tableau III), même durant la saison

TABLEAU III

Évolution de la teneur en phosphore du dactyle.

En g par kg de matière sèche.

\begin{tabular}{|c|c|c|c|c|c|c|c|c|}
\hline Coupe & Organe & $\mathrm{P}$ & Coupe & Organe & $\mathrm{P}$ & Coupe & Organe & $\mathrm{P}$ \\
\hline I & $\underset{\mathrm{PE}}{\mathrm{L}}+\mathrm{T}$ & $\begin{array}{l}3,40 \\
3,70 \\
3,54\end{array}$ & $3 \mathrm{I}$ & $\begin{array}{l}\mathrm{L} \\
\mathrm{G} \\
\mathrm{PE}\end{array}$ & $\begin{array}{l}3,68 \\
2,96 \\
3,56\end{array}$ & 312 & $\begin{array}{l}\mathrm{I} \\
G \\
\mathrm{PE}\end{array}$ & $\begin{array}{l}3,56 \\
2,86 \\
3,40\end{array}$ \\
\hline \multirow[t]{2}{*}{2} & \multirow{2}{*}{$\begin{array}{l}\mathrm{L} \\
G \\
T \\
F \\
P E\end{array}$} & \multirow{2}{*}{$\begin{array}{l}3,20 \\
1,98 \\
2,79 \\
3,34 \\
2,76\end{array}$} & $3^{2}$ & $\begin{array}{l}\mathrm{L} \\
\mathrm{G} \\
\mathrm{PE}\end{array}$ & $\begin{array}{l}3,8+ \\
3,{ }_{4} 3 \\
3,72\end{array}$ & 313 & $\begin{array}{l}\mathrm{L} \\
\mathrm{G} \\
\mathrm{PE}\end{array}$ & $\begin{array}{l}4,25 \\
3,89 \\
4,18\end{array}$ \\
\hline & & & \multirow{2}{*}{33} & \multirow{2}{*}{$\begin{array}{l}\mathrm{L} \\
\mathrm{G} \\
\mathrm{PE}\end{array}$} & \multirow{2}{*}{$\begin{array}{l}3,83 \\
3,28 \\
3,70\end{array}$} & \multirow{2}{*}{331} & \multirow{2}{*}{$\begin{array}{l}\mathrm{L} \\
\mathrm{G} \\
\mathrm{PE}\end{array}$} & \multirow{2}{*}{$\begin{array}{l}5,30 \\
4,47 \\
5,21\end{array}$} \\
\hline & $\stackrel{\mathrm{L}}{\mathrm{G}}+\mathrm{T}$ & $\begin{array}{l}2,61 \\
I, 85\end{array}$ & & & & & & \\
\hline 3 & $\begin{array}{l}\mathrm{F} \\
\mathrm{PE}\end{array}$ & $\begin{array}{l}2,76 \\
2,10\end{array}$ & I I I & $\begin{array}{l}\mathrm{L} \\
\mathrm{G} \\
\mathrm{PE}\end{array}$ & $\begin{array}{l}4,2 \mathrm{I} \\
3, \mathrm{II} \\
4,06\end{array}$ & 332 & $\begin{array}{l}\mathrm{L} \\
\mathrm{C} \\
\mathrm{PE}\end{array}$ & $\begin{array}{l}4,86 \\
4,61 \\
4,82\end{array}$ \\
\hline I I & $\begin{array}{l}\stackrel{\mathrm{L}}{\mathrm{G}}+\mathrm{T} \\
\mathrm{F} \\
\mathrm{PE}\end{array}$ & $\begin{array}{l}3,40 \\
2,59 \\
2,77 \\
3,24\end{array}$ & II 2 & $\begin{array}{l}\mathrm{L} \\
\mathrm{G} \\
\mathrm{PE}\end{array}$ & $\begin{array}{l}4,05 \\
3,14 \\
3,90\end{array}$ & .333 & $\begin{array}{l}\mathrm{L} \\
\mathrm{G} \\
\mathrm{Pl}\end{array}$ & $\begin{array}{l}4,46 \\
4,18 \\
4,41\end{array}$ \\
\hline I 2 & $\begin{array}{l}\mathrm{L} \\
\mathrm{G} \\
\mathrm{PE}\end{array}$ & $\begin{array}{l}3,83 \\
3, \mathrm{I} 3 \\
3,57\end{array}$ & $\mathrm{II}_{3}$ & $\begin{array}{l}\mathrm{L} \\
\mathrm{G} \\
\mathrm{PE}\end{array}$ & $\begin{array}{l}3,77 \\
3,29 \\
3,64\end{array}$ & I I 2 I & $\begin{array}{l}\mathrm{L} \\
\mathrm{G} \\
\mathrm{PE}\end{array}$ & $\begin{array}{l}4,39 \\
3,44 \\
4,28\end{array}$ \\
\hline 13 & $\begin{array}{l}\mathrm{L} \\
\mathrm{G} \\
\mathrm{PE}\end{array}$ & $\begin{array}{l}3,75 \\
3,21 \\
3,59\end{array}$ & ${ }^{I_{3}} \mathrm{I}$ & $\begin{array}{l}\mathrm{L} \\
\mathrm{G} \\
\mathrm{PE}\end{array}$ & $\begin{array}{l}+, 33 \\
3,29 \\
4,2 \mathrm{I}\end{array}$ & I 122 & $\begin{array}{l}\mathrm{L} \\
\mathrm{G} \\
\mathrm{PL}\end{array}$ & $\begin{array}{l}4,51 \\
3,78 \\
4,37\end{array}$ \\
\hline $2 I$ & $\begin{array}{l}\mathrm{L} \\
\mathrm{G} \\
\mathrm{PI}\end{array}$ & $\begin{array}{l}3,70 \\
3,13 \\
3,60\end{array}$ & ${ }^{1} 3^{2}$ & $\begin{array}{l}\mathrm{L} \\
\mathrm{G} \\
\mathrm{PE}\end{array}$ & $\begin{array}{l}4,6 \mathrm{I} \\
3,56 \\
4,4 \mathrm{I}\end{array}$ & $\begin{array}{lll}1 & 123\end{array}$ & $\begin{array}{l}\mathrm{L} \\
\mathrm{G} \\
\mathrm{PE}\end{array}$ & $\begin{array}{l}3,86 \\
3,08 \\
3,68\end{array}$ \\
\hline 22 & $\begin{array}{l}\mathrm{L} \\
\mathrm{G} \\
\mathrm{PE}\end{array}$ & $\begin{array}{l}3,42 \\
3,20 \\
3,37\end{array}$ & $\mathrm{I}_{33}$ & $\begin{array}{l}\mathrm{L} \\
\mathrm{G} \\
\mathrm{PE}\end{array}$ & $\begin{array}{l}4,32 \\
3,60 \\
4,15\end{array}$ & $\mathrm{I}, 3 \mathrm{~T} 2$ & $\begin{array}{l}\mathrm{I} \\
\mathrm{G} \\
\mathrm{PL}\end{array}$ & $\begin{array}{l}4,67 \\
3,88 \\
4,57\end{array}$ \\
\hline 23 & $\begin{array}{l}\mathrm{L} \\
\mathrm{G} \\
\mathrm{PE}\end{array}$ & $\begin{array}{l}3,51 \\
3,09 \\
3,41\end{array}$ & $31 \mathrm{I}$ & $\begin{array}{l}\mathrm{L} \\
\mathrm{G} \\
\mathrm{PE}\end{array}$ & $\begin{array}{l}3,43 \\
2,90 \\
3,34\end{array}$ & 3 122 & $\begin{array}{l}\mathrm{L} \\
\mathrm{G} \\
\mathrm{PE}\end{array}$ & $\begin{array}{l}3,70 \\
2,91 \\
3,56\end{array}$ \\
\hline
\end{tabular}

chaude. Notons cependant que l'été ayant été très pluvieux, nos résultats ne peuvent infirmer les constatations de nombreux auteurs (Hомв 1953. ODELIEN I946...) trouvant une baisse du taux de phosphore due à la sécheresse.

Ia plante entière s'enrichit donc également en $\mathrm{P}$ au fur et à mesure 

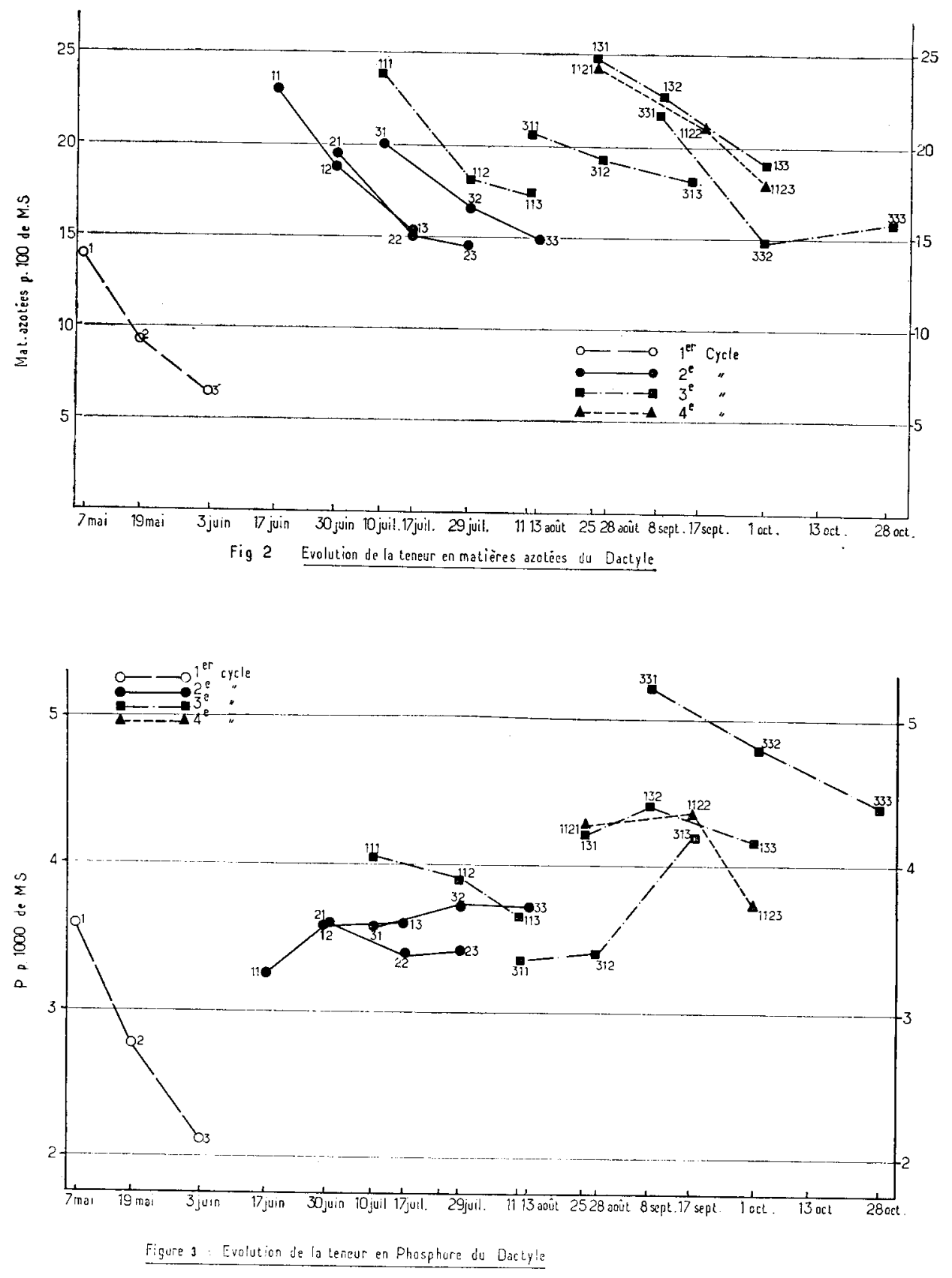
des coupes successives (figure III) d'autant plus que la proportion des limbes, organes les plus riches, devient de plus en plus grande. Les plantes des troisièmes cycles, en particulier les plus tardifs, et $d \mathfrak{u}$ quatrième cycle, sont manifestement les plus riches en $\mathrm{P}$. Ceci peut s'expliquer par l'action favorable des premières pluies d'automne sur la disponibilité de $\mathrm{P}_{2} \mathrm{O}_{5}$ dans le sol et sur la croissance de la plante, et aussi par la proportion importante de feuilles rouillées qui ont dû être éliminées. En effet les composés phosphorés se retireraient des parties mortes de la plante, en automne, et se concentreraient dans les parties vertes ou destinées à survivre (LATSCHAw et MILLER I924). Nous avons nousmêmes constaté que les limbes rouillés sont plus pauvres en $\mathrm{P}$, (teneur moyenne de $\mathrm{I}, 5 \mathrm{~g}$ par $\mathrm{kg}$ ) que les limbes sains (teneur moyenne de $3,5 \mathrm{~g}$ par kg).

\section{Influence du stade de développement.}

Durant le premier cycle, la teneur en $P$ des divers organes, et, par voie de conséquence, la teneur de la plante entière (liée à l'évolution des teneurs des organes et à celle du rapport feuilles/tiges) diminuent considérablement avec le stade de développement, et atteignent des valeurs très faibles à la floraison. Nous savons en effet que la floraison mobilise une quantité importante de phosphore, aux dépens des feuilles en particulier. Ces données corroborent les résultats de nos travaux antérieurs sur le premier cycle du dactyle et de la fétuque.

En revanche, cette constatation faite durant le $\mathrm{I}^{\mathrm{e}}$ cycle, et que l'on a souvent tendance à généraliser, n'est pas toujours valable pour les cycles suivants. En effet, les plantes des cycles suivants ne montent plus en tiges et n'épient plus, aussi elles ne s'appauvrissent que faiblement en $P$ au cours de leur développement,ou tendent même parfois, paradoxalement, à s'enrichir en P. Bien entendu il importe de tenir compte, en particulier pour les $3^{\mathrm{e}}$ et $4^{\mathrm{e}}$ cycles, de la proportion croissante, au fur et à mesure du développement de la plante, des débris rouillés, proportion pouvant atteindre $30 \mathrm{p}$. Ioo de la plante entière au $3^{\mathrm{e}}$ stade des $3^{\mathrm{e}}$ cycles.

\section{$3^{\circ}$ Le Calcium.}

Comme pour le phosphore, les différences de teneurs entre organes, très marquées pour les plantes $\mathrm{du} \mathrm{I}^{\mathrm{e}}$ cycle, se réduisent et s'annulent au cours des cycles suivants. Ainsi, les limbes, bien plus riches en $\mathrm{Ca}$ que les tiges et les gaines pour les premières coupes, présentent par la suite des teneurs voisines de celle: des gaines, et parfois même inférieures (tableau IV). Tout le calcium de la plante peut être obtenu par extraction triple (eau, acide acétique dilué, acide chlorhydrique dilué) mais il est difficile de localiser les différentes formes du calcium dans la plante 
TABLEAU IV

Evolution de la teneur en calcium du dactyle.

En g par kg de matière sèche.

\begin{tabular}{|c|c|c|c|c|c|c|c|c|}
\hline Coupe & Organe & $\mathrm{Ca}$ & Coupe & Organe & $\mathrm{Ca}$ & Coupe & Organe & $\mathrm{Ca}$ \\
\hline I & $\begin{array}{l}\mathrm{L} \\
\mathrm{GE}\end{array}$ & $\begin{array}{l}3,97 \\
2,45 \\
3,24\end{array}$ & $3^{I}$ & $\begin{array}{l}\mathrm{L} \\
\mathrm{G} \\
\mathrm{PE}\end{array}$ & $\begin{array}{l}4,05 \\
3,51 \\
3,95\end{array}$ & $3 \mathrm{I} 2$ & $\begin{array}{l}\mathrm{L} \\
\mathrm{G} \\
\mathrm{PE}\end{array}$ & $\begin{array}{l}3,49 \\
4,07 \\
3,61\end{array}$ \\
\hline \multirow[t]{2}{*}{2} & $\begin{array}{l}\mathrm{L} \\
\mathrm{G} \\
\mathrm{T}\end{array}$ & $\begin{array}{l}4,24 \\
2,23 \\
1,20\end{array}$ & $3^{2}$ & $\begin{array}{l}\mathrm{L} \\
\mathrm{G} \\
\mathrm{PE}\end{array}$ & $\begin{array}{l}3,59 \\
3,65 \\
3,60\end{array}$ & $3^{\mathrm{I}} \hat{3}$ & $\begin{array}{l}\mathbf{L} \\
\mathrm{G} \\
\mathrm{PE}\end{array}$ & $\begin{array}{l}3,57 \\
4,18 \\
3,70\end{array}$ \\
\hline & $\mathrm{PE}$ & 2,23 & \multirow[b]{2}{*}{33} & \multirow{2}{*}{$\begin{array}{l}\mathrm{L} \\
\mathrm{G} \\
\mathrm{PL}\end{array}$} & \multirow{2}{*}{$\begin{array}{l}3,26 \\
3,31 \\
3,27\end{array}$} & \multirow{2}{*}{$33^{\mathbf{I}}$} & \multirow{2}{*}{$\begin{array}{l}\mathrm{L} \\
\mathrm{G} \\
\mathrm{PE}\end{array}$} & \multirow{2}{*}{$\begin{array}{l}5,05 \\
4,75 \\
5,02\end{array}$} \\
\hline \multirow[b]{2}{*}{3} & $\mathrm{~L}$ & 6,64 & & & & & & \\
\hline & $\begin{array}{l}\mathrm{F} \\
\mathrm{PE}\end{array}$ & $\begin{array}{l}1,27 \\
\mathrm{I}, 74 \\
\mathrm{I}, 97\end{array}$ & I I I & $\begin{array}{l}\mathrm{I} \\
\mathrm{G} \\
\mathrm{PE}\end{array}$ & $\begin{array}{l}4,86 \\
4,32 \\
4,79\end{array}$ & $33^{2}$ & $\begin{array}{l}\mathrm{L} \\
\mathrm{G} \\
\mathrm{PE}\end{array}$ & $\begin{array}{l}4,15 \\
3,66 \\
4,08\end{array}$ \\
\hline I I & $\begin{array}{l}\mathrm{L} \\
\mathrm{G} \\
\mathrm{F} \\
\mathrm{PE}\end{array}$ & $\begin{array}{l}4,56 \\
2,87 \\
1,92 \\
4,17\end{array}$ & II 2 & $\begin{array}{l}\mathrm{L} \\
\mathrm{G} \\
\mathrm{PE}\end{array}$ & $\begin{array}{l}4,21 \\
4,6 \mathrm{I} \\
4,27\end{array}$ & 333 & $\begin{array}{l}\mathrm{L} \\
\mathrm{G} \\
\mathrm{PE}\end{array}$ & $\begin{array}{l}3,14 \\
3,18 \\
3,15\end{array}$ \\
\hline I 2 & $\begin{array}{l}\mathrm{L} \\
\mathrm{G} \\
\mathrm{PE}\end{array}$ & $\begin{array}{l}4,05 \\
3,49 \\
3,82\end{array}$ & $\mathrm{II}_{3}$ & $\begin{array}{l}\mathrm{L} \\
\mathrm{G} \\
\mathrm{P} \mathbf{E}\end{array}$ & $\begin{array}{l}3,35 \\
3,54 \\
3,4^{\circ}\end{array}$ & I I $2 \mathrm{I}$ & $\begin{array}{l}\mathrm{L} \\
\mathrm{G} \\
\mathrm{PE}\end{array}$ & $\begin{array}{l}4,86 \\
4,74 \\
4,84\end{array}$ \\
\hline I3 & $\begin{array}{l}\mathrm{L} \\
\mathrm{G} \\
\mathrm{PE}\end{array}$ & $\begin{array}{l}3,31 \\
2,96 \\
3,22\end{array}$ & $\mathrm{I}_{3} \mathrm{r}$ & $\begin{array}{l}\mathrm{L} \\
\mathrm{G} \\
\mathrm{PE}\end{array}$ & $\begin{array}{l}5,07 \\
4,84 \\
5,04\end{array}$ & I 122 & $\begin{array}{l}\mathrm{L} \\
\mathrm{G} \\
\mathrm{PE}\end{array}$ & $\begin{array}{l}3,40 \\
4,09 \\
3,53\end{array}$ \\
\hline 21 & $\begin{array}{l}\mathrm{L} \\
\mathrm{G} \\
\mathrm{PE}\end{array}$ & $\begin{array}{l}4,52 \\
3,71 \\
4,28\end{array}$ & $13^{2}$ & $\begin{array}{l}\mathrm{L} \\
\mathrm{G} \\
\mathrm{PE}\end{array}$ & $\begin{array}{l}4,03 \\
4,47 \\
4, \mathrm{II}\end{array}$ & $\begin{array}{lll}\text { I } & 23\end{array}$ & $\begin{array}{l}\mathrm{L} \\
\mathrm{G} \\
\mathrm{PE}\end{array}$ & $\begin{array}{l}3,64 \\
3,35 \\
3,57\end{array}$ \\
\hline 22 & $\begin{array}{l}\mathrm{L} \\
\mathrm{G} \\
\mathrm{PE}\end{array}$ & $\begin{array}{l}3,36 \\
3,06 \\
3,28\end{array}$ & I33 & $\begin{array}{l}\mathrm{L} \\
\mathrm{G} \\
\mathrm{PE}\end{array}$ & $\begin{array}{l}3,66 \\
3,71 \\
3,67\end{array}$ & 1312 & $\begin{array}{l}\mathrm{L} \\
\mathrm{G} \\
\mathrm{PE}\end{array}$ & $\begin{array}{l}5,03 \\
3,72 \\
4,86\end{array}$ \\
\hline 23 & $\begin{array}{l}\mathrm{L} \\
\mathrm{G} \\
\mathrm{PE}\end{array}$ & $\begin{array}{l}3, \mathrm{I} 4 \\
3,3 \mathrm{I} \\
3, \mathrm{I} 8\end{array}$ & $3^{3 \mathrm{II}}$ & $\begin{array}{l}\mathrm{L} \\
\mathrm{G} \\
\mathrm{PE}\end{array}$ & $\begin{array}{l}4,52 \\
3,49 \\
4,34\end{array}$ & 3122 & $\begin{array}{l}\mathrm{L} \\
\mathrm{G} \\
\mathrm{PE}\end{array}$ & $\begin{array}{l}3,46 \\
3,57 \\
3,4^{8}\end{array}$ \\
\hline
\end{tabular}

(Kostytschew et Berg I929, cités par Baumeister). Cependant la majeure partie du calcium soluble dans l'eau se trouve dans les organes supérieurs et jeunes de la plante (BAuMEISTER I958), le calcium soluble dans les acides (carbonate, phosphate, oxalate) se trouvant surtout localisé dans les parties basses, celles-ci étant par ailleurs les plus pauvres en calcium total. Le calcium jouerait d'autre part un rôle important dans la formation des parois cellulaires sous forme de composés de l'acide pectique.

A stade de développement équivalent (même durée de croissance.) l'influence du cycle de végétation sur les teneurs en $\mathrm{Ca}$ des différents 
organes est très nette : la teneur en $\mathrm{Ca}$ augmente régulièrement au cours de l'année. Par suite, la teneur en $\mathrm{Ca}$ de la plante entière saine s'accroît également, la différence étant particulièrement marquée, comme pour le phosphore, entre le premier cycle et les suivants (figure IV).

$\mathrm{I}_{1}$ influence du stade de développement sur la teneur en $\mathrm{Ca}$ des feuilles de diverses plantes a été étudiée par de nombreux auteurs montrant que les feuilles s'enrichissent constamment en Ca durant la période de végétation, et FrEX-WISSLING (I935, cité par BAUMEISTER) constate que le rapport $\mathrm{K} / \mathrm{Ca}$ décroît considérablement en fonction du vieillissement des feuilles. En fait ces constatations ne sont valables que pour le premier cycle du dactyle, et, au cours des cycles suivants, limbes et gaines s'appauvrissent régulièrement en $\mathrm{Ca}$ en vieillissant. L'influence du stade de développement sur la teneur en $\mathrm{Ca}$ de la plante entière est régulière et très marquée, et se traduit, quel que soitle cycle, par un appauvrissement continu en calcium. Ceci s'explique, pour le premier cycle, par la décroissance brutale du rapport feuilles/tiges (les feuilles étant plus riches que les tiges) dont l'effet ne peut être compensé par l'enrichissement des feuilles.

Il semble exister une étroite corrélation entre l'évolution des taux de calcium et de matières azotées. En revanche, la corrélation définie entre $P$ et les matières azotées au cours $d u$ premier cycle, n'est plus valable à partir du second cycle.

L'influence générale des coupes successives se traduit par un accroissement de la teneur en $\mathrm{Ca}$ de la plante, constatation en accord avec STEWART et Holmes (I953). La mobilité du calcium dans la plante serait très variable (BAUMEISTER I958) et il n'y aurait pas de migration à partir des feuilles jaunissantes en automne. Nous avons constaté que les parties rouillées des limbes étaient très légèrement plus riches en calcium que les parties saines.

\section{$4^{\circ}$ Le Potassium.}

Les différences de teneurs entre organes ne varient pas d'un cycle à l'autre, et sont d'ailleurs peu accentuées. Cependant les gaines sont en général mieux pourvues en $\mathrm{K}$ que les limbes (tableau $\mathrm{V}$ ).

A stade de développement équivalent les teneurs en $\mathrm{K}$ des divers organes diminuent au cours de l'année, malgré un accroissement très net et passager probablement dû̀ à une action climatique à la mi-été (figure V), ce qui est contraire aux constatations de STEWART et HoLMes (I953) qui trouvent un minimum à cette saison par suite d'un climat sans doute différent. Il en résulte que la teneur en $\mathrm{K}$ de la plante entière saine diminue aussi considérablement au cours de l'année, malgré le passage du potassium des parties rouillées vers les parties vertes. Nous 


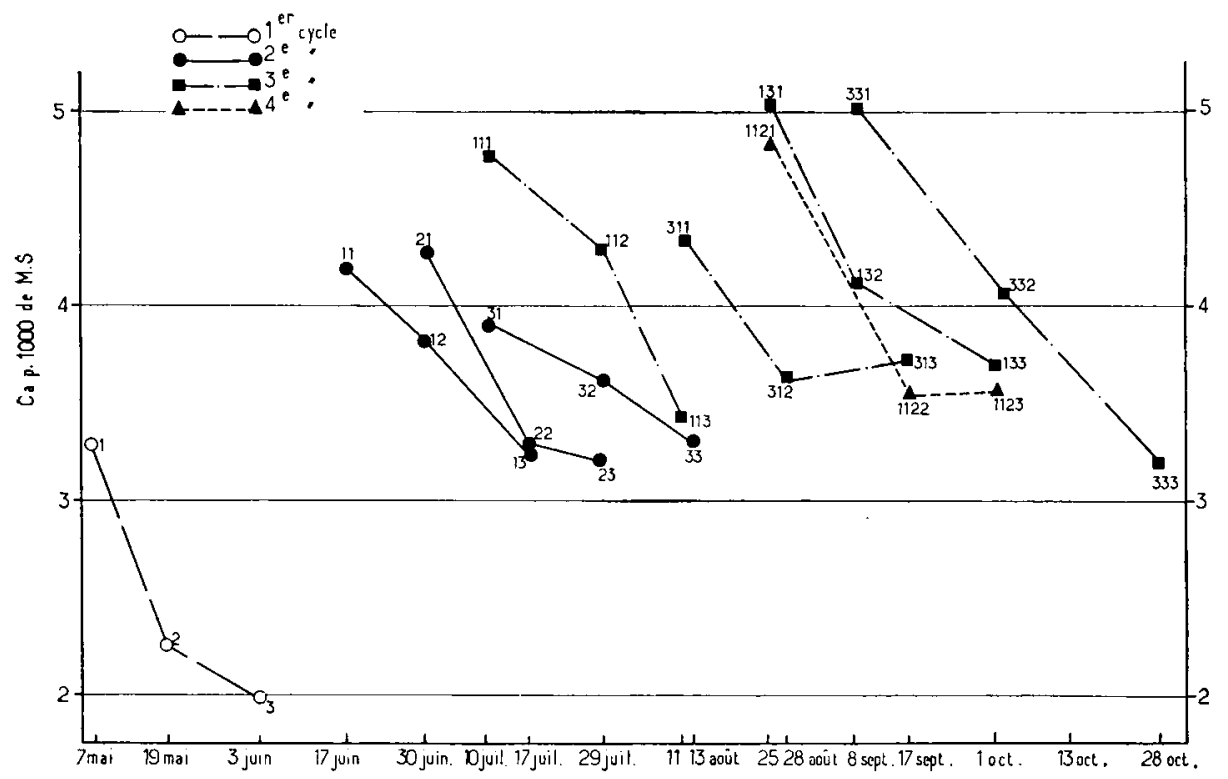

Figure \& Evolution de la teneur en Calcium du Dactyle

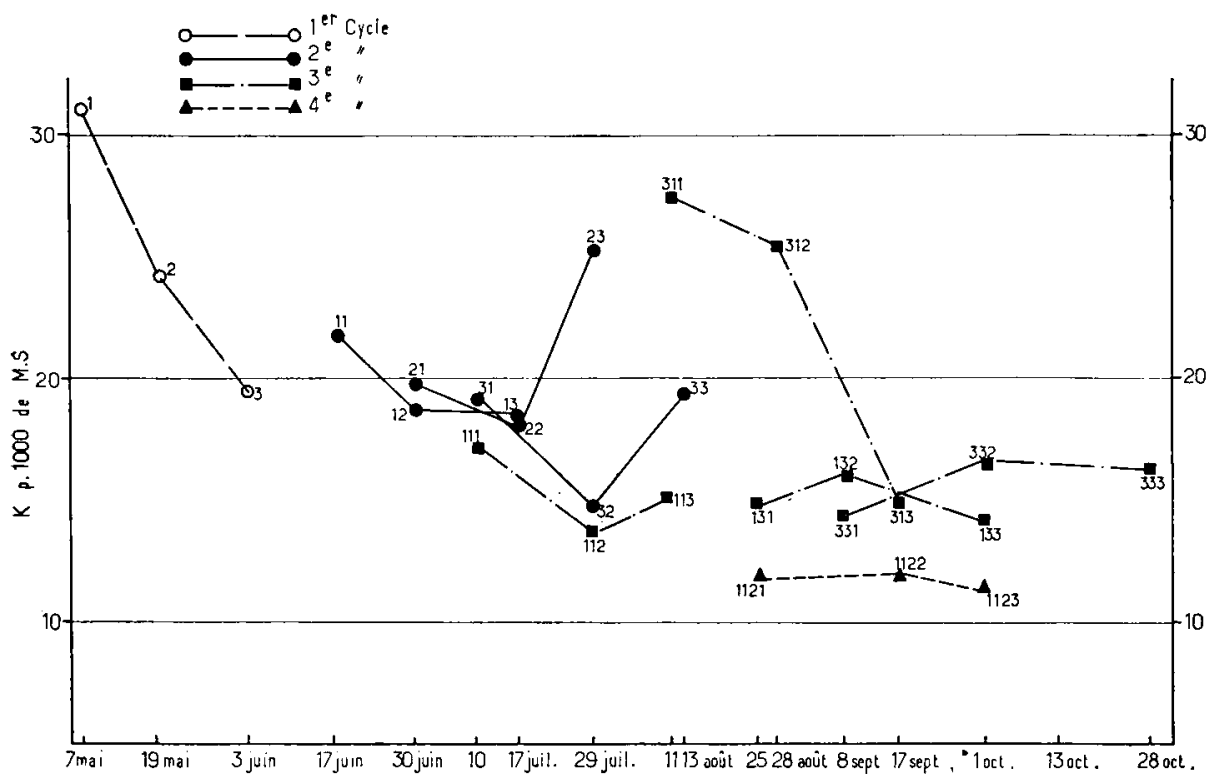

Figure 3 Evolution de la teneur en potassium du Dactyle 
TABIEAU V

Evolution de la teneur en potassium du dactyle.

En $g$ par $k g$ de matière sèche.

\begin{tabular}{|c|c|c|c|c|c|c|c|c|}
\hline Coupe & Organe & $\mathrm{K}$ & Coupe & Organe & $\mathrm{K}$ & Coupe & Organe & $\mathrm{K}$ \\
\hline I & $\begin{array}{l}\mathrm{L} \\
\mathrm{PE}\end{array}+\mathrm{T}$ & $\begin{array}{l}28,25 \\
33,5 \\
30,75\end{array}$ & $3 I$ & $\begin{array}{l}\mathrm{L} \\
\mathrm{G} \\
\mathrm{PE}\end{array}$ & $\begin{array}{l}18,71 \\
22,43 \\
19,34\end{array}$ & $3^{12}$ & $\begin{array}{l}\mathrm{L} \\
\mathrm{G} \\
\mathrm{PE}\end{array}$ & $\begin{array}{l}24,18 \\
30,00 \\
25,45\end{array}$ \\
\hline 2 & & $\begin{array}{l}26,74 \\
24,25 \\
25,49\end{array}$ & 32 & $\begin{array}{l}\mathrm{L} \\
\mathrm{G} \\
\mathrm{PE}\end{array}$ & $\begin{array}{l}15,00 \\
13,44 \\
14,73\end{array}$ & $3^{1} 3$ & $\begin{array}{l}\mathrm{L} \\
\mathrm{G} \\
\mathrm{PE}\end{array}$ & $\begin{array}{l}15,27 \\
\text { I3,08 } \\
\text { I4,82 }\end{array}$ \\
\hline & $\mathrm{PE}$ & $24,3^{2}$ & & $\mathrm{~L}$ & 19,25 & & $\mathrm{~L}$ & 14,10 \\
\hline & $\stackrel{\mathrm{L}}{\mathrm{G}}+\mathrm{T}$ & 22,6 & 33 & $\mathrm{PE}$ & 19,37 & $30^{1}$ & $\mathrm{PE}$ & 14,38 \\
\hline 3 & $\begin{array}{l}\mathrm{F} \\
\mathrm{PE}\end{array}$ & $\begin{array}{l}10,56 \\
19,4^{\circ}\end{array}$ & III I & $\begin{array}{l}\mathrm{L} \\
\mathrm{G} \\
\mathrm{PE}\end{array}$ & $\begin{array}{l}16,73 \\
20,32 \\
17,22\end{array}$ & $33^{2}$ & $\begin{array}{l}\mathrm{L} \\
\mathrm{G} \\
\mathrm{PE}\end{array}$ & $\begin{array}{l}\text { I } 6,36 \\
\text { I } 7,94 \\
\text { г } 6,58\end{array}$ \\
\hline $\mathrm{X} \mathbf{I}$ & $\begin{array}{l}\mathrm{L} \\
\mathrm{G} \\
\mathrm{F} \\
\mathrm{PE}\end{array}$ & $\begin{array}{r}21,35 \\
27,16 \\
9,40 \\
21,78\end{array}$ & I I 2 & $\begin{array}{l}\mathrm{L} \\
\mathrm{G} \\
\mathrm{PE}\end{array}$ & $\begin{array}{l}14,16 \\
10,93 \\
13,64\end{array}$ & 3.33 & $\begin{array}{l}\mathrm{L} \\
\mathrm{G} \\
\mathrm{PE}\end{array}$ & $\begin{array}{l}\mathrm{I} 7,43 \\
\mathrm{II}, 47 \\
\mathrm{I} 6,2 \mathrm{I}\end{array}$ \\
\hline $\mathrm{I} 2$ & $\begin{array}{l}\mathrm{L} \\
\mathrm{G} \\
\mathrm{PE}\end{array}$ & $\begin{array}{l}18,49 \\
22,67 \\
\text { I } 8,64\end{array}$ & I I 3 & $\begin{array}{l}\mathrm{L} \\
\mathrm{G} \\
\mathrm{PE}\end{array}$ & $\begin{array}{l}15,04 \\
15,35 \\
15,12\end{array}$ & I I $2 \mathrm{I}$ & $\begin{array}{l}\mathrm{L} \\
\mathrm{G} \\
\mathrm{PE}\end{array}$ & $\begin{array}{l}\mathrm{I} 1,47 \\
\mathrm{I} 3,25 \\
\mathrm{I}, 67\end{array}$ \\
\hline 13 & $\begin{array}{l}\mathrm{L} \\
\mathrm{G} \\
\mathrm{PE}\end{array}$ & $\begin{array}{l}\text { I } 8,30 \\
\text { I9,04 } \\
18,49\end{array}$ & ${ }^{1} 3^{I}$ & $\begin{array}{l}\mathrm{L} \\
\mathrm{G} \\
\mathrm{PE}\end{array}$ & $\begin{array}{l}14,59 \\
15,67 \\
14,71\end{array}$ & 1122 & $\begin{array}{l}\mathrm{L} \\
\mathrm{G} \\
\mathrm{PE}\end{array}$ & $\begin{array}{r}\mathrm{I} 2,74 \\
8,5 \mathrm{I} \\
\mathrm{I}, 93\end{array}$ \\
\hline $2 \mathrm{I}$ & $\begin{array}{l}\mathrm{L} \\
\mathrm{G} \\
\mathrm{PE}\end{array}$ & $\begin{array}{l}19,37 \\
21,55 \\
\mathrm{I} 9,75\end{array}$ & $\mathrm{I}^{32}$ & $\begin{array}{l}\mathrm{L} \\
\mathrm{G} \\
\mathrm{PE}\end{array}$ & $\begin{array}{l}15,94 \\
16,40 \\
16,0,3\end{array}$ & 1123 & $\begin{array}{l}\mathrm{L} \\
\mathrm{G} \\
\mathrm{PE}\end{array}$ & $\begin{array}{r}\mathrm{Ir}, 96 \\
8,54 \\
\mathrm{II}, \mathrm{I} 7\end{array}$ \\
\hline 22 & $\begin{array}{l}\mathrm{L} \\
\mathrm{G} \\
\mathrm{PE}\end{array}$ & $\begin{array}{l}18,20 \\
16,91 \\
17,89\end{array}$ & 133 & $\begin{array}{l}\mathrm{L} \\
\mathrm{G} \\
\mathrm{PE}\end{array}$ & $\begin{array}{l}14,64 \\
12,07 \\
14,06\end{array}$ & 1312 & $\begin{array}{l}\mathrm{L} \\
\mathrm{G} \\
\mathrm{PE}\end{array}$ & $\begin{array}{l}\text { I } 4,36 \\
\text { I } 0,79 \\
\text { I } 3,88\end{array}$ \\
\hline 23 & $\begin{array}{l}\mathrm{L} \\
\mathrm{G} \\
\mathrm{PE}\end{array}$ & $\begin{array}{l}24,80 \\
27,05 \\
25,34\end{array}$ & $3 \mathrm{II}$ & $\begin{array}{l}\mathrm{L} \\
\mathrm{G} \\
\mathrm{PE}\end{array}$ & $\begin{array}{l}25,92 \\
34,38 \\
27,38\end{array}$ & 3122 & $\begin{array}{l}\mathrm{L} \\
\mathrm{G} \\
\mathrm{PE}\end{array}$ & $\begin{array}{l}26,28 \\
30,24 \\
27,01\end{array}$ \\
\hline
\end{tabular}

savons en effet que le potassium est essentiellement intracellulaire et lié aux processus vitaux, et en fait, nous constatons également que les parties rouillées des limbes sont particulièrement pauvres en $\mathrm{K}$ (teneur moyenne de $3 \mathrm{~g}$ par $\mathrm{kg}$ ).

$\mathrm{I}_{\mathrm{a}}$ teneur en potassium diminue considérablement avec le stade de développement au cours du premier cycle, dans les divers organes en particulier dans les tiges, et par suite dans la plante entière.

Au cours des périodes particulièrement pluvieuses (août I958) les taux de $\mathrm{K}$ dans la plante augmentent considérablement (figure $\mathrm{V}$ ). Ceci semble dû à une action favorable sur 1'absorption de $\mathrm{K}$ par la plante. 
Selon Morris et SAyre (I935) tout le potassium de la plante se trouve probablement sous forme ionisée et est totalement extractible par l'eau froide.

En conclusion, on peut remarquer que, contrairement aux autres éléments étudiés, le potassium est beaucoup plus abondant dans les plantes du I er cycle.

\section{$5^{\circ}$ Le Sodium.}

On remarque tout d'abord que les teneurs en sodium du dactyle étudié sont très élevées, en particulier pour les derniers cycles. Ceci confirme nos résultats récents (GUEGUEN I959) sur un autre dactyle cultivé

\section{Tableau VI}

Evolution de la teneur en sodium du dactyle. En g par kg de matière sèche.

\begin{tabular}{|c|c|c|c|c|c|c|c|c|}
\hline Coupe & Organe & $\mathrm{Na}$ & Coupe & Organe & $\mathrm{Na}$ & Coupe & Organe & $\mathrm{Na}$ \\
\hline I & $\underset{\mathrm{PE}}{\mathrm{L}}+\mathrm{T}$ & $\begin{array}{l}2,44 \\
2,45 \\
2,44\end{array}$ & $3 \mathbf{I}$ & $\begin{array}{l}\mathrm{L} \\
\mathrm{G} \\
\mathrm{PE}\end{array}$ & $\begin{array}{r}7,57 \\
10,68 \\
8, \mathrm{rI}\end{array}$ & $3 \mathrm{I} 2$ & $\begin{array}{l}\mathrm{L} \\
\mathrm{G} \\
\mathrm{PE}\end{array}$ & $\begin{array}{l}5,76 \\
9,67 \\
6,60\end{array}$ \\
\hline \multirow[t]{2}{*}{2} & $\begin{array}{l}\mathrm{L} \\
\mathrm{G} \\
\mathrm{T}\end{array}$ & $\begin{array}{l}3, \mathrm{I} 2 \\
2,32 \\
2,99\end{array}$ & $3^{2}$ & $\begin{array}{l}\mathrm{L} \\
\mathrm{P} \\
\mathrm{E}\end{array}$ & $\begin{array}{r}8,39 \\
\text { Io,25 } \\
8,63\end{array}$ & $3^{\mathrm{I}} 3$ & $\begin{array}{l}\mathrm{L} \\
\mathrm{G} \\
\mathrm{PE}\end{array}$ & $\begin{array}{l}8,16 \\
9,00 \\
8,35\end{array}$ \\
\hline & $\mathrm{PE}$ & $2,4^{2}$ & \multirow{2}{*}{33} & \multirow{2}{*}{$\begin{array}{l}\mathrm{L} \\
\mathrm{G} \\
\mathrm{PE}\end{array}$} & \multirow{2}{*}{$\begin{array}{l}7,02 \\
9,83 \\
7,70\end{array}$} & \multirow{2}{*}{$33 \mathrm{I}$} & \multirow{2}{*}{$\begin{array}{l}\mathrm{L} \\
\mathrm{G} \\
\mathrm{PE}\end{array}$} & \multirow{2}{*}{$\begin{array}{r}8,84 \\
\mathbf{1} 3,85 \\
8,76\end{array}$} \\
\hline & $\mathrm{L}$ & 3,28 & & & & & & \\
\hline 3 & $\begin{array}{l}\mathrm{F}+1 \\
\mathrm{PE}\end{array}$ & $\begin{array}{l}2,52 \\
0,10 \\
2,08\end{array}$ & I I I & $\begin{array}{l}\mathrm{L} \\
\mathrm{G} \\
\mathrm{PE}\end{array}$ & $\begin{array}{l}\text { Io,56 } \\
\text { I, } 6,54 \\
\text { I } 1,39\end{array}$ & $33^{2}$ & $\begin{array}{l}\mathrm{L} \\
\mathrm{G} \\
\mathrm{PE}\end{array}$ & $\begin{array}{r}6,89 \\
10,32 \\
7,38\end{array}$ \\
\hline I I & $\begin{array}{l}\mathrm{L} \\
\mathrm{G} \\
\mathrm{F} \\
\mathrm{PE}\end{array}$ & $\begin{array}{l}4,92 \\
4,77 \\
0,12 \\
4,55\end{array}$ & $\mathrm{II} 2$ & $\begin{array}{l}\mathrm{L} \\
\mathrm{G} \\
\mathrm{PE}\end{array}$ & $\begin{array}{r}9,36 \\
12,32 \\
9,83\end{array}$ & 333 & $\begin{array}{l}\mathrm{L} \\
\mathrm{G} \\
\mathrm{PE}\end{array}$ & $\begin{array}{r}7, \mathrm{I} 7 \\
\mathrm{II}, 25 \\
7,98\end{array}$ \\
\hline 12 & $\begin{array}{l}\mathrm{L} \\
\mathrm{G} \\
\mathrm{PE}\end{array}$ & $\begin{array}{r}6,45 \\
10,40 \\
6,94\end{array}$ & $\mathrm{II}_{3}$ & $\begin{array}{l}\mathrm{L} \\
\mathrm{G} \\
\mathrm{PE}\end{array}$ & $\begin{array}{r}8,50 \\
13,08 \\
9,70\end{array}$ & I I $2 \mathrm{I}$ & $\begin{array}{l}\mathrm{L} \\
\mathrm{G} \\
\mathrm{PE}\end{array}$ & $\begin{array}{l}\mathrm{I} 0,92 \\
\mathrm{I} 6,64 \\
\mathrm{II}, 57\end{array}$ \\
\hline$I_{3}$ & $\begin{array}{l}\mathrm{I} \\
\mathrm{G} \\
\mathrm{PE}\end{array}$ & $\begin{array}{r}7,43 \\
\text { I0,28 } \\
8, \mathrm{I} 4\end{array}$ & $I_{3} \mathrm{I}$ & $\begin{array}{l}\mathrm{L} \\
\mathrm{G} \\
\mathrm{PE}\end{array}$ & $\begin{array}{r}9,33 \\
15,25 \\
10,01\end{array}$ & I I 22 & $\begin{array}{l}\mathrm{L} \\
\mathrm{G} \\
\mathrm{PE}\end{array}$ & $\begin{array}{l}\mathrm{II}, 28 \\
\mathrm{I} 7,76 \\
\mathrm{I} 2,5 \mathrm{I}\end{array}$ \\
\hline $2 \mathrm{I}$ & $\begin{array}{l}\mathrm{L} \\
\mathrm{G} \\
\mathrm{PE}\end{array}$ & $\begin{array}{r}6,8 \mathbf{I} \\
\mathbf{1 0 , 8 2} \\
8,03\end{array}$ & 132 & $\begin{array}{l}\mathrm{L} \\
\mathrm{G} \\
\mathrm{PE}\end{array}$ & $\begin{array}{r}9,80 \\
16,25 \\
\text { I I,02 }\end{array}$ & $\mathrm{I} I 23$ & $\begin{array}{l}\mathrm{L} \\
\mathrm{G} \\
\mathrm{PE}\end{array}$ & $\begin{array}{r}9,62 \\
\text { I } 3,91 \\
10,60\end{array}$ \\
\hline 22 & $\begin{array}{l}\mathrm{L} \\
\mathrm{G} \\
\mathrm{PE}\end{array}$ & $\begin{array}{l}7,07 \\
9,46 \\
7,64\end{array}$ & $\mathbf{I} 33$ & $\begin{array}{l}\mathrm{I} \\
\mathrm{G} \\
\mathrm{PE}\end{array}$ & $\begin{array}{l}\text { I0,i6 } \\
\text { I4,04 } \\
\text { I } 1,05\end{array}$ & I 312 & $\begin{array}{l}\mathrm{L} \\
\mathrm{G} \\
\mathrm{PE}\end{array}$ & $\begin{array}{l}\text { I1 }, 88 \\
\text { I } 5,62 \\
\text { I } 2,37\end{array}$ \\
\hline 23 & $\begin{array}{l}\mathrm{L} \\
\mathrm{G} \\
\mathrm{PE}\end{array}$ & $\begin{array}{l}4,47 \\
6,45 \\
4,94\end{array}$ & $3^{\mathrm{rI}}$ & $\begin{array}{l}\mathrm{L} \\
\mathrm{G} \\
\mathrm{PE}\end{array}$ & $\begin{array}{r}7,04 \\
\text { Io,or } \\
7,54\end{array}$ & 3122 & $\begin{array}{l}\mathrm{L} \\
\mathrm{G} \\
\mathrm{PE}\end{array}$ & $\begin{array}{l}5,80 \\
9,41 \\
6,47\end{array}$ \\
\hline
\end{tabular}




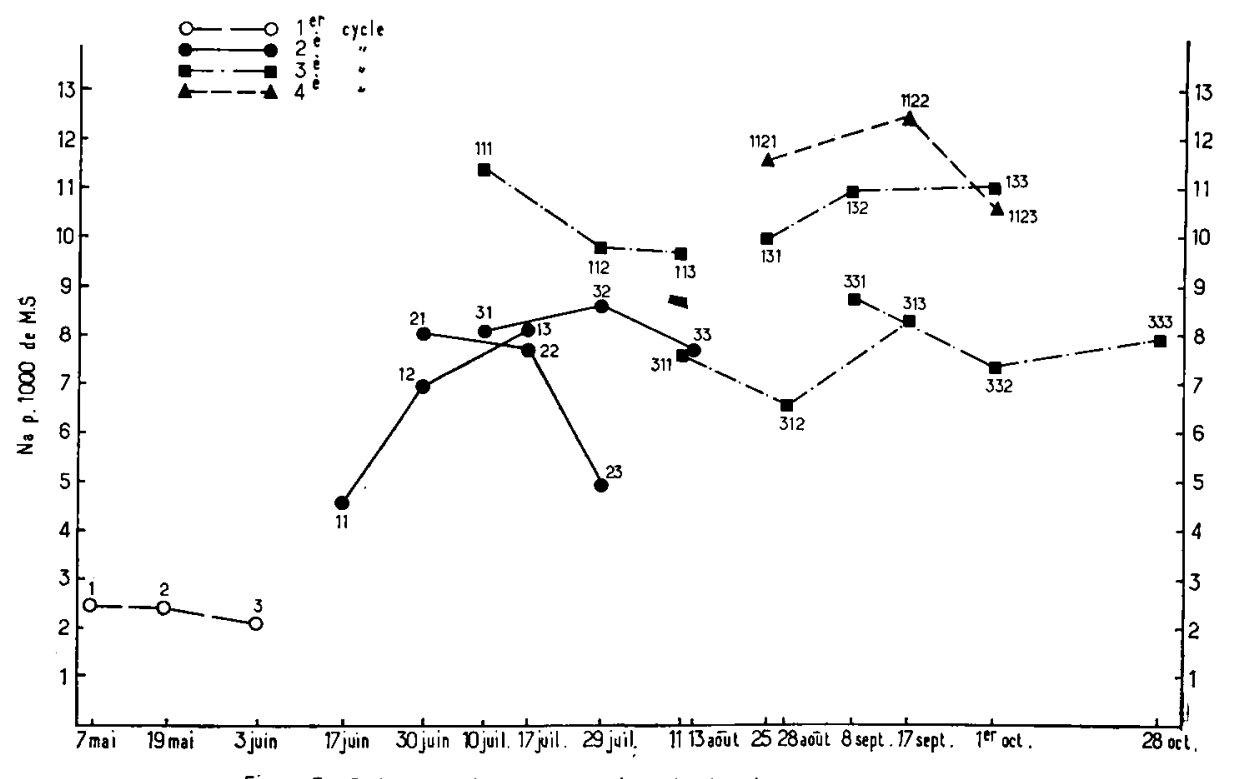

Figure 6 : Evolution de la teneur en sodium du dactyle

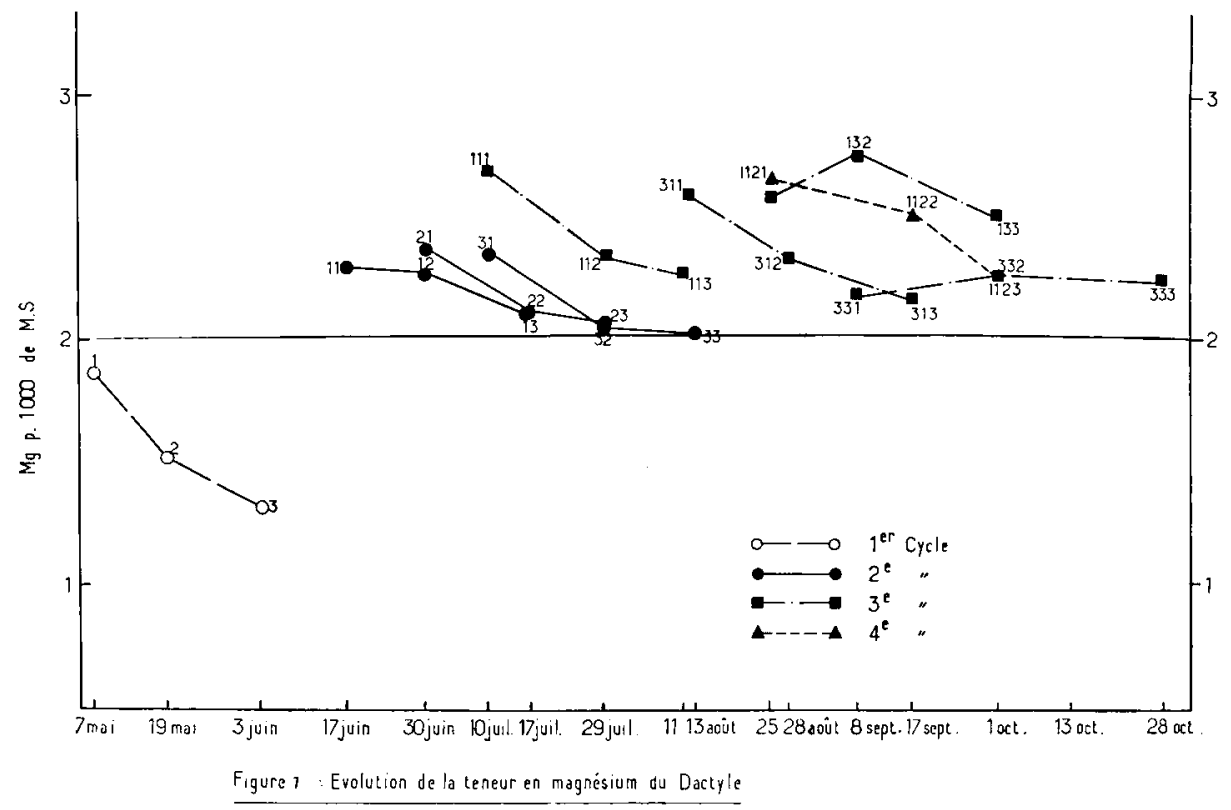


sur le même type de sol. A notre connaissance de telles teneurs n'ont été citées que par STEWART et Holmes, trouvant des teneurs de 8 à Io $\mathrm{g}$ de $\mathrm{Na}$ par $\mathrm{kg}$ de matière sèche sur des graminées cultivées à proximité de la mer. En fait, il existe peu de données analytiques sur les dernières coupes d'un dactyle pur, et d'autre part les teneurs en sodium semblent varier considérablement suivant la nature du sol (les plantes poussant sur sol calcaire étant en général pauvres en sodium, d'après R.tDET I958) et aussi d'après la souche même de dactyle.

Quel que soit le cycle de végétation, les gaines sont nettement plus riches en sodium que les limbes (tableau VI). A stade botanique

\section{TABleau VII}

Évolution de la teneur en magnésium du dactyle.

En g par kg de matière sèche.

\begin{tabular}{|c|c|c|c|c|c|c|c|c|}
\hline Coupe & Organe & $\mathrm{Mg}$ & Coupe & Organe & $\mathrm{Mgg}$ & Coupe & Organe & $\mathrm{MI}_{\mathrm{r} r}$ \\
\hline I & $\underset{\mathrm{PE}}{\mathrm{L}}+\mathrm{T}$ & $\begin{array}{l}\mathrm{T}, 85 \\
\mathrm{r}, 9 \mathrm{r} \\
\mathrm{I}, 8 \mathrm{7}\end{array}$ & $3^{\mathrm{I}}$ & $\begin{array}{l}\mathrm{L} \\
\mathrm{G} \\
\mathrm{PE}\end{array}$ & $\begin{array}{l}2,36 \\
2,45 \\
2,37\end{array}$ & $3^{12}$ & $\begin{array}{l}\mathrm{L} \\
\mathrm{G} \\
\mathrm{P} E\end{array}$ & $\begin{array}{l}2,28 \\
2,56 \\
2,34\end{array}$ \\
\hline 2 & $\begin{array}{l}\mathrm{L} \\
\mathrm{G} \\
\mathrm{T} \\
\mathrm{F}\end{array}$ & $\begin{array}{l}2,07 \\
1,34 \\
\mathrm{I}, 42\end{array}$ & 32 & $\begin{array}{l}\mathrm{L} \\
\mathrm{G} \\
\mathrm{PE}\end{array}$ & $\begin{array}{l}2,05 \\
2,08 \\
2,05\end{array}$ & $3^{1} 3$ & $\begin{array}{l}\mathrm{L} \\
\mathrm{G} \\
\mathrm{PE}\end{array}$ & $\begin{array}{l}2,12 \\
2,35 \\
2,16\end{array}$ \\
\hline & $\mathrm{PE}$ & 1,52 & & $\mathrm{~L}$ & 2,03 & 33 & $\mathrm{~L}$ & 2,13 \\
\hline & $\underset{\mathrm{G}}{\mathrm{L}}+\mathrm{T}$ & $\begin{array}{l}2,77 \\
1,13\end{array}$ & 3,3 & $\mathrm{PE}$ & 2,03 & $30^{2}$ & $\mathrm{PE}$ & 2,18 \\
\hline 3 & $\begin{array}{l}\mathrm{F} \\
\mathrm{PE}\end{array}$ & $\begin{array}{l}1,16 \\
1,32\end{array}$ & III & $\begin{array}{l}\mathrm{I} \\
\mathrm{G} \\
\mathrm{PE}\end{array}$ & $\begin{array}{l}2,66 \\
2,99 \\
2,70\end{array}$ & 332 & $\begin{array}{l}\mathrm{L} \\
\mathrm{G} \\
\mathrm{PE}\end{array}$ & $\begin{array}{l}2,29 \\
2,11 \\
2,27\end{array}$ \\
\hline II & $\begin{array}{l}\mathrm{L} \\
\mathrm{G}+\mathrm{T} \\
\mathrm{F} \\
\mathrm{PE}\end{array}$ & $\begin{array}{l}2,39 \\
2,21 \\
1,08 \\
2,30\end{array}$ & 112 & $\begin{array}{l}\mathrm{I} \\
\mathrm{G} \\
\mathrm{PE}\end{array}$ & $\begin{array}{l}2,34 \\
2,37 \\
2,34 \\
\end{array}$ & 333 & $\begin{array}{l}\mathrm{L} \\
\mathrm{G} \\
\mathrm{P} E\end{array}$ & $\begin{array}{l}2,24 \\
2,23 \\
2,24 \\
\end{array}$ \\
\hline I 2 & $\begin{array}{l}\mathrm{L} \\
\mathrm{G} \\
\mathrm{PL}\end{array}$ & $\begin{array}{l}2,35 \\
2,41 \\
2,28\end{array}$ & $I_{3}$ & $\begin{array}{l}\mathrm{L} \\
\mathrm{G} \\
\mathrm{PE}\end{array}$ & $\begin{array}{l}2,25 \\
2,33 \\
2,27\end{array}$ & I I 21 & $\begin{array}{l}\mathrm{L} \\
\mathrm{G} \\
\mathrm{PL}\end{array}$ & $\begin{array}{l}2,62 \\
3,01 \\
2,66\end{array}$ \\
\hline I3 & $\begin{array}{l}\mathrm{L} \\
\mathrm{G} \\
\mathrm{P} \mathrm{L}\end{array}$ & $\begin{array}{l}2,32 \\
2,26 \\
2,10\end{array}$ & $I_{3} \mathrm{I}$ & $\begin{array}{l}\mathrm{L} \\
\mathrm{G} \\
\mathrm{PE}\end{array}$ & $\begin{array}{l}2,56 \\
2,77 \\
2,59 \\
\end{array}$ & I I 22 & $\begin{array}{l}\mathrm{L} \\
\mathrm{G} \\
\mathrm{PE}\end{array}$ & $\begin{array}{l}2,50 \\
2,61 \\
2,52 \\
\end{array}$ \\
\hline $2 \mathrm{I}$ & $\begin{array}{l}\mathrm{L} \\
\mathrm{G} \\
\mathrm{I}^{\prime} \mathrm{E}\end{array}$ & $\begin{array}{l}2,41 \\
2,31 \\
2,38\end{array}$ & 1.32 & $\begin{array}{l}\mathrm{L} \\
\mathrm{G} \\
\mathrm{PE}\end{array}$ & $\begin{array}{l}2,66 \\
2,76 \\
2,67\end{array}$ & I 123 & $\begin{array}{l}\mathrm{L} \\
\mathrm{G} \\
\mathrm{PE}\end{array}$ & $\begin{array}{l}2,30 \\
2,12 \\
2,26\end{array}$ \\
\hline 22 & $\begin{array}{l}\mathrm{I} \\
\mathrm{G} \\
\mathrm{P}^{\prime} \mathrm{E}\end{array}$ & $\begin{array}{l}2,17 \\
1,98 \\
2,12\end{array}$ & I 33 & $\begin{array}{l}\mathrm{L} \\
\mathrm{G} \\
\mathrm{PE}\end{array}$ & $\begin{array}{l}2,54 \\
2,35 \\
2,50\end{array}$ & $13^{12}$ & $\begin{array}{l}\mathrm{L} \\
\mathrm{G} \\
\mathrm{PE}\end{array}$ & $\begin{array}{l}2,80 \\
2,45 \\
2,75 \\
\end{array}$ \\
\hline 23 & $\begin{array}{l}\mathrm{L} \\
\mathrm{G} \\
\mathrm{PE}\end{array}$ & $\begin{array}{l}2,06 \\
2,1,3 \\
2,07\end{array}$ & 3II & $\begin{array}{l}\mathrm{L} \\
\mathrm{G} \\
\mathrm{PE}\end{array}$ & $\begin{array}{l}2,58 \\
2,74 \\
2,60\end{array}$ & 3122 & $\begin{array}{l}\mathrm{L} \\
\mathrm{G} \\
\mathrm{I} \mathbf{E}\end{array}$ & $\begin{array}{l}2,43 \\
2,6 \mathrm{I} \\
2,4^{6}\end{array}$ \\
\hline
\end{tabular}


équivalent, les divers organes s'enrichissent généralement en sodium au cours de la saison. De même, le taux de Na de la plante entière augmente considérablement à mesure que le nombre de coupes croît (figure VI).

Ie stade de développement exerce une influence très irrégulière sur le taux de sodium de la plante, et ce fait ne permet pas de dégager une règle générale.

En accord avec STEWART et HoLMES (I953) nous notons une tendance nette vers une diminution de la teneur en sodium au milieu de l'été cependant particulièrement pluvieux (correspondant en revanche à des teneurs élevés en $\mathrm{K}$ ).

\section{$6^{\circ}$ Le Magnésium.}

L'évolution du magnésium dans la plante est parallèle à celle du calcium, l'amplitude des variations étant moins forte.

Comme pour le calcium, les limbes sont les organes les plus riches au cours du premier cycle; par contre, au cours des cycles suivants, ils sont fréquemment plus pauvres que les gaines (tableau VII). Les plantes entières du premier cycle sont beaucoup plus pauvres en $\mathrm{Mg}$ que celles des cycles suivants. D'autre part, à partir du second cycle, la teneur en Mg de la plante entière ne varie que faiblement (figure VII).

L'influence du stade de développement, moins accentuée que pour le calcium, se traduit par une diminution régulière de la teneur en $\mathrm{Mg}$ de la plante entière et des différents organes.

Notons que l'élimination des parties rouillées ne peut influer considérablement sur le taux de $\mathrm{Mg}$ dans la plante entière, car le magnésium de la chlorophylle ne représente que ro à $20 \%$ du magnésium total et les limbes rouillés ne sont pas plus pauvres en Mg que les limbes verts.

\section{III. - DISCUSSION.}

\section{Conséquences pour l'alimentation des animaux.}

Après avoir comparé les besoins en éléments minéraux des animaux (en particulier des vaches laitières) aux teneurs trouvées dans le dactyle à différentes périodes de l'année, nous essayerons de définir, dans notre cas particulier (année $195^{8}$ pluvieuse) le mode d'exploitation. à préconiser pour réaliser l'apport optimum de matières azotées et d'éléments minéraux dans l'alimentation des animaux.

Io Comparaison des besoins et des apports

pour les différents cycles de végétation du dactyle.

Seul le premier cycle présente rapidement des teneursen matières azotées (inférieures à I $40 \mathrm{~g}$ par $\mathrm{kg}$ de matière sèche) pouvant constituer un facteur 
limitant pour les animaux exigeants (vaches laitières fortes productrices).

En ce qui concerne les besoins en phosphore, nous constatons que seules les coupes tardives du premier cycle sont insuffisantes pour assurer une production laitière moyenne. En effet, utilisé au début de l'épiaison par une vache de $600 \mathrm{~kg}$, le dactyle étudié ne permet pas une production supérieure à $\mathrm{I} 5$ litres de lait, et, au début de la floraison, il ne permet plus une production de ro litres de lait. Par contre, et quel que soit le stade de développement, les plantes récoltées au cours des autres cycles de végétation permettraient d'assurer les plus fortes productions laitières (teneurs en $\mathrm{P}$ supérietures à $3,3 \mathrm{~g}$ par $\mathrm{kg}$ de matière sèche).

Les teneurs en calcium au contraire, sont en général insuffisantes pour couvrir les besoins des vaches bonnes productrices et seuls les pâturages précoces à partir du $3^{\mathrm{e}}$ cycle peuvent permettre de fortes productions laitières sans apport de complément. Les plantes âgées du premier cycle sont particulièrement carencées en calcium (teneurs inférieures à $2,5 \mathrm{~g}$ de $\mathrm{Ca}$ par $\mathrm{kg}$ de matière sèche). Cependant, quel que soit le cycle de végétation, une exploitation normale du dactyle par les animaux (30-40 jours de croissance) nécessiterait l'usage de compléments calciques.

En ce qui concerne le potassium, il est bien connu que les teneurs rencontrées dans les fourrages sont largement excédentaires, et, comme le montrent nos résultats, ceci est particulièrement vrai pour le dactyle du I er cycle, qui est également le seul cycle où la plante s'appauvrit en $\mathrm{K}$ en vieillissant. En revanche, les coupes suivantes sont beaucoup moins riches en $\mathrm{K}$; étant donné les inconvénients possibles que présente un excès de potassium (pouvant nuire à l'utilisation digestive d'autres éléments minéraux, et en particulier du magnésium) ces coupes semblent mieux convenir à l'alimentation rationnelle des animaux.

Les besoins en sodium sont de 2 à $3 \mathrm{~g}$ par $\mathrm{kg}$ de matière sèche consommée, pour une vache moyenne productrice dans des conditions normales. Cependant ces besoins peuvent atteindre $6 \mathrm{~g}$ de $\mathrm{Na}$ par $\mathrm{kg}$ de matière sèche pour une vache recevant seulement de l'herbe jeune. Nous constatons que pratiquement toutes les repousses de dactyle sont suffisamment pourvues en sodium pour couvrir les besoins accrus par un régime de pâturage. Cette constatation, si elle se confirmait dans d'autres conditions de sol et de climat, serait particulièrement importante, puisqu'elle enlèverait tout l'intérêt d'une distribution de sel aux vaches laitières pâturant des repousses de dactyle.

L'apport de magnésium par le dactyle est suffisant pour couvrir les besoins des vaches laitières. Cependant les plantes du premier cycle sont nettement les plus pauvres en $\mathrm{Mg}$, et, compte tenu du gros apport de potassium correspondant, il est prudent, pour prévenir les accidents tétaniques éventuels, de complémenter un pâturage de printemps par l'apport d'un sel de magnésium. 


\section{$2^{\circ}$ Recherche du mode d'exploitation à préconiser} pour le dactyle étudié.

Compte tenu des différences de composition minérale observées entre les différents cycles, des rendements en matière sèche obtenus pour les diverses coupes (figure $\mathrm{I}$ ) et des variations de la teneur en matières azotées, il doit être possible de définir le ou les modes d'exploitation permettant d'assurer un bon apport azoté et minéral dans l'alimentation des animaux, tout en fournissant en quantité satisfaisante un fourrage de bonne valeur nutritive bien consommé par les animaux.

Si les dernières coupes sont particulièrement riches en éléments minéraux intéressants, les conditions climatiques ne permettent plus, en revanche, d'obtenir des rendements et des consommations satisfaisants, d'autant plus que la proportion des feuilles malades et non consommées devient très importante. Il importe donc de choisir le deuxième cycle de végétation, ou au plus tard un troisième cycle très précoce, pour récolter le maximum de fourrage de bonne valeur nutritive. A cet effet, il est nécessaire d'interrompre précocement le premier cycle de telle façon que la montaison ne puisse plus se produire au cours du cycle suivant, c'est-à-dire en sectionnant la plante plus bas que l'inflorescence. Comme la valeur nutritive des plantes du premier cycle diminue très rapidement avec le stade de développement, il est recommandé de les utiliser au stade le plus précoce permettant de sectionner les épis dans la gaine. Il sera enstuite loisible, pour le cycle suivant, d'atteindre les plus hauts rendements sans craindre une diminution importante de la valeur nutritive de l'herbe, pour ce qui concerne les matières azotées et les éléments minéraux.

Des travaux récents de Minson et HARRIs (I958) montrent par ailleurs que la digestibilité de la matière sèche du dactyle diminue très rapidement après le début de l'épiaison au cours du premier cycle, et beaucoup moins vite au cours du second cycle. Ainsi, en I958, la digestibilité du dactyle a diminué au cours du second cycle de 73,6 à 63 p. Ioo de la $4^{\mathrm{e}}$ à la $8^{\mathrm{e}}$ semaine de croissance, tandis qu'elle diminuait de 74 à $57 \mathrm{p}$. roo au cours du premier cycle, durant les 4 semaines suivant le début de l'épiaison. Au stade normal d'exploitation en foin du second cycle, correspondant à 6-7 semaines de croissance, la digestibilité est encore de $66 \mathrm{p}$. Ioo environ.

\section{SUMMARY}

We have studied the fluctuations in the content of nitrogenous material and mineral elements (P, Ca, K, Na, Mg) of a forage grass, cocksfoot, (Dactylis glomerata), after separating the plant into its different components, leaf laminas, leaf sheaths, stems, inflorescences and debris; this also enables changes in the composition of the various organs to be followed. 
The experimental plots were subdivided in order to obtain several vegetative cycles arising from a previous cycle which had been interrupted at various stages of development; the advantages and disadvantages of several methods of management can thus be studied. In each of the cycles studied, the cocksfoot was harvested at three different developmental stages.

The nitrogenous matter $(\mathrm{N} \times 6.25)$ was determined by the Kjeldah1 method. The mineral elements were determined, after ashing the sample : Phosphorus by a colorimetric, ammonium phosphovanado-molybdate method, and calcium, sodium, potassium and magnesium by flame spectrophotometry.

In general, the first vegetative cycle differs markedly from the following cycles, both as regards the actual chemical composition of the plant and its different organs, and as regards the changes in composition with age of the plant. These differences are primarily due to morphological dissimilarities between plants, since stem elongation and flowering do not generally occur again after the first cycle.

The differences in the contents between plant parts, important in the first cycle when the leaf laminas are generally richest, become negligible in the succeeding cycles, when the leaf laminas and leaf sheaths have a similar composition, except for their nitrogenous matter.

At an equivalent developmental stage, the plants of the last cycles are generally better supplied with $\mathrm{P}, \mathrm{Ca}, \mathrm{Na}$ and $\mathrm{Mg}$ whose contents increase regularly during the year. On the other hand, potassium is most abundant in the plants of the first cycle and its content falls in the following cycles.

The influence of the stage of development on the nitrogen, calcium and magnesium content of the cocksfoot is very distinct, whatever the vegetative cycle : their content decreases regularly with the age of the plant. But as regards the phosphorus and potassium contents, only the plants of the first cycle show a marked reduction, the composition of the plants of the following cycles being little modified by the developmental stage. This is particularly important for the phosphorus content of the plant, which falls consiclerably, but only in the first cycle, a phenomenon which one tends to attribute to the following cycles. Finally, the age of the plant has a very irregular effect on the sodium content, which in the cocksfoot studied is particularly high from the second cycle onwards.

By comparing the needs of animals for each of the elements studied with the contents found in the plants of the different cycles, and bearing in mind the variation in the dry-matter yield, one can define the method of management to be recommended.

It is advantageous to replace the first cycle by the second whose plants have a higher nutritive value (with regard to nitrogen and minerals) without an important loss in yield; this is obtained in practice by cutting or grazing relatively early in the first cycle (before ear-emergence) so that the inflorescences are cut off when still in their sheaths.

\section{RÉFERRENCES BIBLIOGRAPHIQUES}

Baumeister W., 1958. " Handbuch der Pflanzen Physiologie ". W. Ruhland. Springer-Verlag-Berlin, p. $482-557$.

Fauconneau G. et Jarrige R., I957. Composition chimique et valeur nutritive de 1'herbe. Butl. Tech. Ing. Ser. agric., 118. 
GUeGUen I., I959. Étude des variations de la composition minérale de quelques espèces fourragères. Influence du stade de développement et đu cycle de végétation. Ann. Zootech., 8, 245-268.

Gueguen L. ; et Rombauts P., ig60. Dosage du sodium, du potassium, du calcium et du magnésium par spectrophotométrie de flamme dans les aliments, le lait et les excreta. Ann. Biol. Anim. Bioch. et Biophys., 1, (sous presse).

Jarrige R., I956. Bull Tech. Ing. Ser. Agric. (Hors série).

LAtschaw I. L. et Militer E. C., I924. Elemental composition of the com plant. J. Agric. Res, 2\%, 845-860.

Maynard L. A. et Loosli K. J., I956. Animal Nutrition. Mc Graw-Hill Book company Inc. New-York, IOS-I35.

Minson D. J. et Harris C. E., I959, Experiments in Progress. The Grassland Research Institute. Hurley.

Morris V. H. et SAYre J. D., I935. Solubility of potassium in corn tissues. Plant Physiol., 10, 565-568.

Morrison F. B., I954. "Feeds and Feeding ". 2 I st. ed. Morrison pub. Co. Ithaca N. Y. Appendix.

RADET E., I958. 'Travaux de la Station d'Agronomie de la Marne.

STEWART A. B., Holimes W., I953. Manuring of grassland. I. Some effects of heavy dressings of nitrogen on the mineral composition of grassland Herbage. J. Sci. Food A gric., 9, $401-408$. 